\title{
Spatiotemporal Filter for Visual Motion Integration from Pursuit Eye Movements in Humans and Monkeys
}

\author{
죽ishna Mukherjee, ${ }^{1}$ Bing Liu, ${ }^{1}$ C.Claudio Simoncini, ${ }^{1}$ and ${ }^{-L e s l i e ~ C . ~ O s b o r n e ~}{ }^{1,2}$ \\ ${ }^{1}$ Department of Neurobiology and ${ }^{2}$ Department of Organismal Biology and Anatomy, The University of Chicago, Chicago, Illinois 60637
}

\begin{abstract}
Despite the enduring interest in motion integration, a direct measure of the space-time filter that the brain imposes on a visual scene has been elusive. This is perhaps because of the challenge of estimating a 3D function from perceptual reports in psychophysical tasks. We take a different approach. We exploit the close connection between visual motion estimates and smooth pursuit eye movements to measure stimulus-response correlations across space and time, computing the linear space-time filter for global motion direction in humans and monkeys. Although derived from eye movements, we find that the filter predicts perceptual motion estimates quite well. To distinguish visual from motor contributions to the temporal duration of the pursuit motion filter, we recorded single-unit responses in the monkey middle temporal cortical area (MT). We find that pursuit response delays are consistent with the distribution of cortical neuron latencies and that temporal motion integration for pursuit is consistent with a short integration MT subpopulation. Remarkably, the visual system appears to preferentially weight motion signals across a narrow range of foveal eccentricities rather than uniformly over the whole visual field, with a transiently enhanced contribution from locations along the direction of motion. We find that the visual system is most sensitive to motion falling at approximately one-third the radius of the stimulus aperture. Hypothesizing that the visual drive for pursuit is related to the filtered motion energy in a motion stimulus, we compare measured and predicted eye acceleration across several other target forms.
\end{abstract}

Key words: linear analysis; motion perception; sensory-motor behavior; smooth pursuit

\section{Significance Statement}

A compact model of the spatial and temporal processing underlying global motion perception has been elusive. We used visually driven smooth eye movements to find the 3D space-time function that best predicts both eye movements and perception of translating dot patterns. We found that the visual system does not appear to use all available motion signals uniformly, but rather weights motion preferentially in a narrow band at approximately one-third the radius of the stimulus. Although not universal, the filter predicts responses to other types of stimuli, demonstrating a remarkable degree of generalization that may lead to a deeper understanding of visual motion processing.

\section{Introduction}

Differences in motion across space are highly informative about the structure of a visual scene. For example, spatially localized motion discontinuities differentiate objects from background

Received Aug. 23, 2016; revised Dec. 3, 2016; accepted Dec. 10, 2016.

Author contributions: T.M., B.L., and L.C.O. designed research; T.M., B.L., C.S., and L.C.O. performed research;

T.M., B.L., C.S., and L.C.O. analyzed data; T.M. and L.C.0. wrote the paper.

This work was supported by grants the Alfred P Sloan Foundation (L.C.O.), the Whitehall Foundation (L.C.O.), the Brain Research Foundation (L.C.O.), the National Institutes of Health (National Eye Institute Grant EY023371 to L.C.O.), and the National Science Foundation (Grant IOS 145704 to L.C.O.). We thank the members of the Osborne laboratory for general assistance with data collection and helpful conversations and the veterinary staff of the Animal Resources Center at University of Chicago.

The authors declare no competing financial interests.

Correspondence should be addressed to Leslie C. Osborne, Department of Neurobiology, University of Chicago, 947 E 58th Street, Abbott 307, Chicago IL 60637. E-mail: osborne@uchicago.edu.

DOI:10.1523/JNEUROSCI.2682-16.2016

Copyright $\odot 2017$ the authors $\quad 0270-6474 / 17 / 371394-19 \$ 15.00 / 0$
(Braddick, 1993) and the distribution of motion vectors across the visual field form a cue for heading direction (Gibson, 1950). By reconfiguring how motion signals are weighted across space, the brain could extract motion contrast or sum motion across a portion of the visual field to subserve different perceptions and actions as needed. Here, we focus on global motion summation. Much of what we know about motion summation comes from analysis of perceptual thresholds for detection or discrimination (van Doorn and Koenderink, 1984; de Bruyn and Orban, 1988; Watamaniuk and Sekuler, 1992; Fredericksen and Verstraten, 1994; Watamaniuk and Heinen, 1999; Tadin et al., 2003; Tadin and Lappin, 2005). Thresholds represent net signal-to-noise and thus make it challenging to recover the underlying weighting of motion signals across space and time. Correlation analyses can differentiate spatial (Neri and Levi, 2009) or temporal (Tadin et al., 2006) contributions to perceptual motion estimates, but the enormous data samples involved limit sampling resolution. Our 
goal is to analyze global motion processing jointly in space and time at high resolution, so we take a different experimental approach. We used smooth pursuit eye tracking as a proxy for the brain's internal estimate of motion direction. We created spatial and temporal variation in motion direction and correlated motion at different locations and time lags with the eye direction. We find the linear model that best predicts pursuit, and we explored how the filter changes with stimulus size and form. We show that the filter reflects visual summation that is shared with perception rather than visual-motor processing specific to pursuit (Krauzlis and Adler, 2001; Wilmer and Nakayama, 2007).

The visual system determines many features of the initial pursuit response. The initial eye acceleration is performed open loop, driven by feedforward visual motion estimates without extraretinal feedback (Lisberger and Westbrook, 1985; Movshon et al., 1990). Visual estimates for pursuit and perception arise in the extrastriate middle temporal cortical area (MT), where neurons respond selectively to visual motion and are tuned for direction and speed (Maunsell and Van Essen, 1983; Newsome et al., 1985; Newsome and Pare, 1988; Komatsu and Wurtz, 1989; Britten et al., 1996; Groh et al., 1997; Lisberger and Movshon, 1999; Rudolph and Pasternak, 1999; Born et al., 2000; Pack and Born, 2001; Hohl and Lisberger, 2011). For motion that drives pursuit well, downstream motor processing of the visual signal adds little noise such that stimulus motion is faithfully translated into eye movement (Osborne et al., 2005; Osborne et al., 2007; Lisberger, 2010; Mukherjee et al., 2015). Under these conditions, the eye movement is correlated both with perceptual estimates of stimulus motion (Stone and Krauzlis, 2003; Mukherjee et al., 2015) and with motion-sensitive cortical neuron responses (Groh et al., 1997; Pack and Born, 2001; Hohl and Lisberger, 2011; Hohl et al., 2013). Taken together, these properties suggest that pursuit can be a good proxy for system-level estimates of visual motion. Indeed, we find that the spatiotemporal filter for pursuit predicts perceptual judgments of motion direction.

Pursuit responds both to the motion of small objects and to spatially distributed motion such as random dot kinematograms (Lisberger et al., 1987). In this sense, pursuit bridges two different scales of spatial motion processing, one operating on local motion contrast and another that sums motion over larger visual regions. In theory, motion contrast and motion summation require different cortical processing and therefore might be modeled by different spatiotemporal filters (Born and Tootell, 1992; Born et al., 2000). To focus on global motion integration, we employ high-contrast, dot motion stimuli that are well suited to the correlation analysis, drive a robust pursuit response, and require motion signals to be pooled across space and time to estimate the direction of travel. We also explore the filter's dependence on stimulus size and form.

\section{Materials and Methods}

Procedures. All experimental procedures adhered to the guidelines of the University of Chicago's Institutional Review Board and Institutional Animal Care and Use Committee and were in strict compliance with the National Institutes of Health's Guide for the Care and Use of Laboratory Animals.

Data acquisition. We recorded eye movements from four adult male rhesus monkeys that had substantial prior training on pursuit tasks. Not all monkeys participated in all experiments. Monkeys viewed stimuli in a dimly lit room on a Sony GDM-FW9011 fast CRT display (display mode 100 frames/s (fps), $1024 \times 768$ pixels) subtending $57^{\circ} \times 38^{\circ}$ at a viewing distance of $48 \mathrm{~cm}$ or a Dell P1130 ( $100 \mathrm{fps}, 1024 \times 768)$ subtending $35^{\circ} \times$ $32^{\circ}$ at a viewing distance of $51 \mathrm{~cm}$. We measured the horizontal and vertical position of one eye with an implanted scleral coil (Robinson,
1963). Eye position was sampled at $1 \mathrm{~ms}$ intervals, vertical and horizontal eye velocity signals were passed through a double-pole, low-pass filter that differentiated frequencies $<25 \mathrm{~Hz}$ and rejected higher frequencies with a roll-off of $20 \mathrm{~dB}$ per decade to yield velocity signals that were then stored for later analysis (Mukherjee et al., 2015).

We also recorded eye movements in three adult (one male, two female) human subjects who gave informed consent. The human subjects had normal or corrected to normal vision and had some prior experience in psychophysical and eye-tracking tasks. Not all subjects participated in all experiments. We measured the vertical and horizontal positions of the right eye with a dual Purkinje image infrared eye tracker (Ward ElectroOptics Gen 6). Eye positions were sampled every $1 \mathrm{~ms}$ and then filtered and stored using the same electronics described above. Subjects viewed stimuli in a dimly lit room on a Sony GDM-FW900 fast CRT display $(100 \mathrm{fps}, 1024 \times 768$ pixels $)$ subtending $35^{\circ} \times 21^{\circ}$ at a viewing distance of $68 \mathrm{~cm}$.

Pursuit experiments. Pursuit experiments were organized into trials lasting 2-3 s that consisted of an initial fixation period of random duration $(700-1400 \mathrm{~ms})$, a pursuit period $(960 \mathrm{~ms})$, and a final fixation period $(400 \mathrm{~ms})$ (Fig. $1 \mathrm{~A})$. The fixation target was a small $\left(0.25^{\circ}\right.$ or $\left.0.5^{\circ}\right)$, uniformly illuminated circle. The fixation target extinguished as a pursuit target appeared synchronously with motion onset. Pursuit targets appeared either centered on the fixation location or $2-3^{\circ}$ eccentric from it and then translated back toward the former fixation point in the classic step-ramp experimental design that minimizes the frequency of saccades (Rashbass, 1961). Pursuit experiments with spatiotemporal noise targets (described below) had motion within a stationary aperture for $160 \mathrm{~ms}$ to initiate pursuit, after which the pattern and aperture translated together for the remaining pursuit period (Fig. 1A). The "motion-within" period allowed calculation of the spatiotemporal filter based on dot motion without contribution from aperture translation. The translation period maintained a high pursuit gain throughout the experiment. Monkeys were rewarded at the end of each successfully completed trial. Human subjects did not receive performance feedback. Both monkeys and humans were required to maintain fixation within $2^{\circ}$ of a stationary spot for $700 \mathrm{~ms}$ before motion onset and to be within $3^{\circ}$ of the target center during the final $200 \mathrm{~ms}$ of pursuit. We expanded the accuracy windows for large targets as needed. We did not penalize gaze accuracy during time windows used for data analysis. To minimize anticipatory eye movements, we randomized the initial fixation interval duration by $700 \mathrm{~ms}$ and we balanced the directions of target motion about the center. Target directions ranged from $-6^{\circ}$ to $+6^{\circ}$ from rightward or leftward in $3^{\circ}$ increments except where noted. We used one target form for each day's experiment. We presented stimuli in blocks rather than interleaving target forms based on our observation that pursuit movements reach a steady-state gain when the target form is expected, whereas alternating target forms can cause strong stimulus history effects in eye speed (Heinen et al., 2005). Monkeys performed $\sim 1500-3000$ trials per daily session. Humans typically performed $\sim 300$ trials per session, so we pooled multiple sessions to reach the necessary sample size. We inspected each trial record and discarded trials with saccades or blinks during the analysis window. We collected data until we accumulated $\sim 100$ repetitions of each experimental condition for analysis.

Perceptual discrimination experiments. We performed a series of perceptual motion discrimination experiments in two of the human subjects for whom we recorded pursuit $(\mathrm{H} 1, \mathrm{H} 3)$. The perceptual discrimination experiments used the same noisy dot direction stimuli as the pursuit tasks. We configured the perceptual experiments as two-alternativeforced-choice (2AFC) tasks. Subjects reported whether the timeaveraged center-of-mass motion direction was upward or downward with respect to horizontal $\left(0^{\circ}\right)$. For these experiments, we presented only rightward directions $\left(0^{\circ}, \pm 6^{\circ}, \pm 12^{\circ}\right)$. Perceptual trials began with a randomized fixation interval $(500-1000 \mathrm{~ms})$. Then, a stationary, $30^{\circ}$ circular diameter random dot pattern appeared on screen for $500 \mathrm{~ms}$, after which the dots moved (see description of noisy dot direction stimuli) within the stationary aperture for $250 \mathrm{~ms}$ (Fig. 1C). Subjects then reported motion direction by button press in a $1250 \mathrm{~ms}$ period. The purpose of the $500 \mathrm{~ms}$ stationary interval was to minimize the contribution of visual activity driven by stimulus appearance rather than motion. 
A

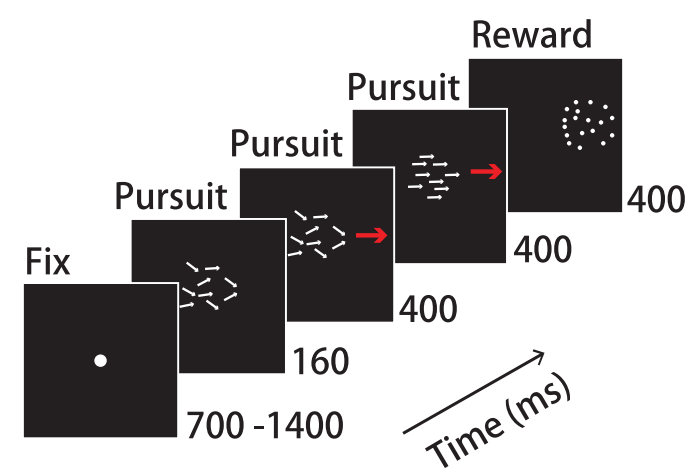

C

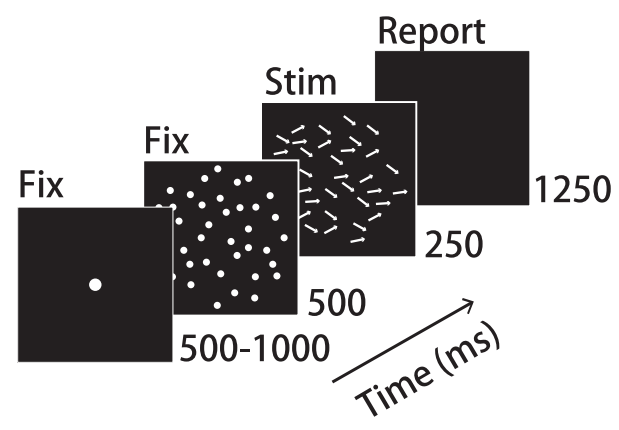

B

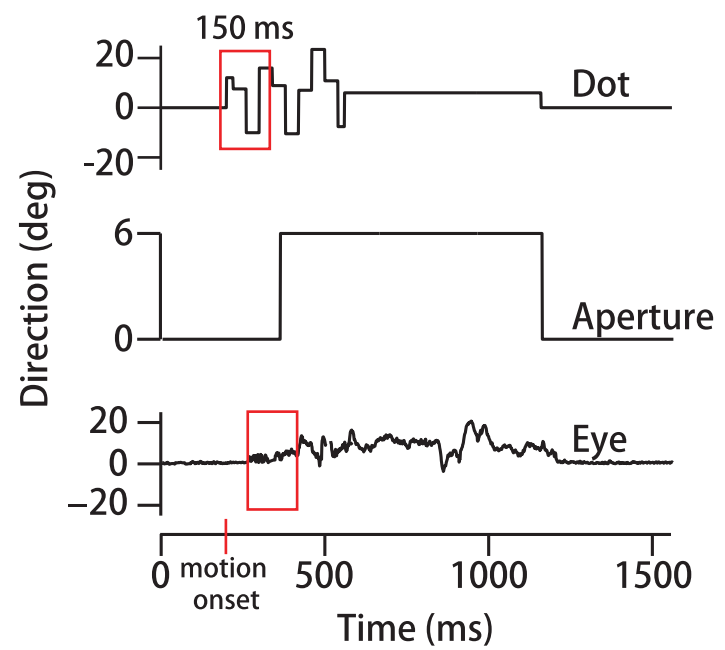

D

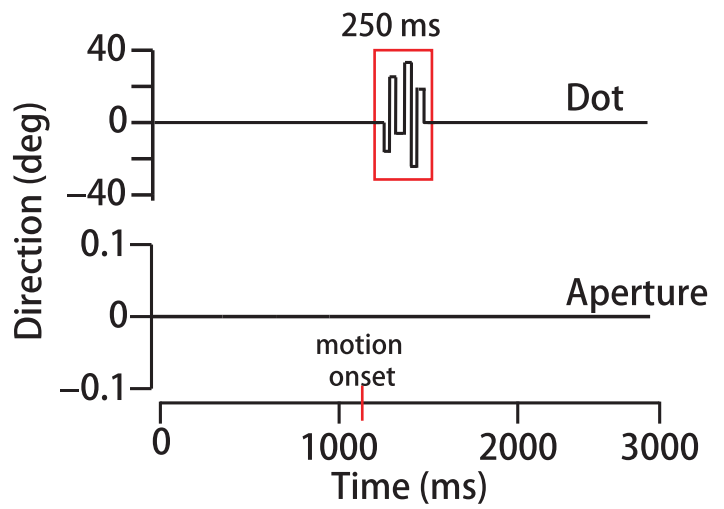

Figure 1. Experimental design. $\boldsymbol{A}$, Schematic representation of a step-ramp pursuit trial sequence. Stimuli consisted of 3-pixel "dots" randomly positioned within a circular aperture. $\boldsymbol{B}$, Top, Direction of a single dot in a noisy dot stimulus over time during the pursuit task. Each dot's direction fluctuated randomly within $\pm 40^{\circ}$ of the mean, updated synchronously every $40 \mathrm{~ms}$. Middle, Aperture motion over time. Dots moved within a stationary aperture for $160 \mathrm{~ms}$ before both translated together across the screen at $15^{\circ} / \mathrm{s}$. Bottom, Eye direction over time on an example trial. The red boxes represent the $150 \mathrm{~ms}$ analysis windows. C, Schematic representation of the perceptual task. After a $500 \mathrm{~ms}$ interval during which the dot pattern was stationary, the dots underwent a step change in speed, moving behind a stationary $30^{\circ}$-diameter aperture for $250 \mathrm{~ms}$. Human subjects then had $1250 \mathrm{~ms}$ to indicate their up/down judgments of the average motion direction by button press. D, Top, Direction of a single dot in the noisy dot stimulus (40 ms update) over time. Red box indicates the $250 \mathrm{~ms}$ analysis window. Bottom, Aperture did not translate in the perception experiment.

Neurophysiology experiments. To measure neural filters for motion integration, we recorded extracellularly from well isolated units in MT of two adult male rhesus monkeys (Macaca mulatta) that had performed pursuit tasks (M2, M3). We implanted a recording chamber, head restraint, and eye coil under anesthesia using sterile technique. Monkeys maintained fixation during physiology experiments to stabilize stimuli in each neuron's receptive field (RF). Extracellular recordings were made with quartz-coated tungsten microelectrodes (TREC). Signals were filtered, amplified, digitized, and recorded through the Plexon OmniPlex system. Initially, we isolated single units using the Plexon online sorter, and we inspected and refined the isolation using Plexon's offline sorter. Once we isolated a unit, we mapped its direction and speed tuning functions and the location and size of its excitatory RF using coherent random dot pattern stimuli. We then created visual stimuli online that had a time-averaged mean direction centered on the flank of the isolated unit's direction tuning curve and that moved at the neuron's preferred speed.

Monkeys were required to maintain fixation of a $0.5^{\circ}$ spot within a $2^{\circ}$ window throughout the trial $(\sim 1-4 \mathrm{~s})$ for a juice reward. Visual stimuli were projected in the RF of the neuron after an initial fixation period of $200 \mathrm{~ms}$. The stimuli remained stationary on screen for another $200 \mathrm{~ms}$ to allow for the neural response to stimulus appearance to decay (Osborne et al., 2004). Stimuli were spatiotemporal noise targets as described below or, more typically, coherent motion dot patterns undergoing randomized direction changes about a constant mean motion direction. In both cases, dot directions were updated every 20 or $40 \mathrm{~ms}$ as in the pursuit experiments and stimuli moved for 500-4000 ms. We recorded 44 MT neurons ( $n=26 \mathrm{M} 2 ; n=18 \mathrm{M} 3$ ) using multiple levels of motion direction variation to yield multiple data points per neuron.

Spatiotemporal noise stimuli. To provide the spatial and temporal variation in motion direction needed to compute a spatiotemporal filter, we added a stochastic component to random dot kinematograms, modeled on stimuli developed by Williams and Sekuler (1984) (see also Heinen and Watamaniuk, 1998; Watamaniuk and Heinen, 1999; Osborne and Lisberger, 2009; Watamaniuk et al., 2011; Mukherjee et al., 2015). The "noisy dots" stimuli comprised high-intensity "dots" (3 pixels) positioned randomly within a circular aperture displayed against the dark background of the CRT monitor (Fig. 1). Aperture diameters ranged from $5^{\circ}$ to $30^{\circ}$. Dot density was $1 \mathrm{dot} / \mathrm{deg}^{2}$ except where noted. Each dot had a common base direction and speed with an individually added stochastic perturbation in direction. Each dot's direction was updated synchronously with a random draw (with replacement) from a uniform direction distribution of $-40^{\circ}$ to $+40^{\circ}$ around the base direction in $1^{\circ}$ increments. The interval between direction updates was $40 \mathrm{~ms}(4$ frames). Between update intervals, each dot moved across the screen 
along a different direction. The noisy dots targets resemble the motion of a swarm of bees or a flock of birds in which each has a random trajectory but there is also overall cohesion in swarm translation. The spatial resolution of our displays limited the actual direction resolution to $\sim 3-6^{\circ}$ between updates. The effects of limited display resolution were not apparent when watching the stimulus or in the movements of the eye even when tracking a single dot. If a dot's updated position fell outside of the aperture, it was repositioned randomly along the opposite edge. For pursuit experiments, the dots moved within a stationary aperture for 160 $\mathrm{ms}$ before dots and aperture began translating together. All targets except for the $30^{\circ}$ noisy dots stimulus had an aperture speed of $15^{\circ} / \mathrm{s}$ for pursuit tasks and base directions of $-6^{\circ},-3^{\circ}, 0,+3^{\circ}$, or $+6^{\circ}$ rightward and leftward. The $30^{\circ}$ diameter noisy dots stimuli had motion within a stationary aperture for the entire duration of the pursuit experiment. Because the direction varies across dots, the apparent translational speed of the dot swarm is slower than that of the aperture. To keep the dot pattern in register with the aperture as they translated, dot speed was increased to $16.4 \%$, a correction equivalent to the SD of the direction distribution (Watamaniuk and Heinen, 1999; Osborne and Lisberger, 2009; Mukherjee et al., 2015).

For human perceptual direction discrimination experiments, we used the same $30^{\circ}$ diameter noisy dot direction stimulus as in the pursuit experiments. The range of dot directions remained $-40^{\circ}$ to $+40^{\circ}$ around rightward base directions of $0^{\circ}, \pm 6^{\circ}$, and $\pm 12^{\circ}$. Dot directions updated synchronously every $40 \mathrm{~ms}$. Dots moved at $16.4^{\circ}$ /s within the stationary aperture.

Coherent motion stimuli for physiology and pursuit. We created coherent motion versions of the noisy dots stimuli to compare temporal motion filters in MT neurons and pursuit. The statistics of direction updates were similar to those for noisy dots $\left(-40^{\circ}\right.$ to $+40^{\circ}$ uniform direction distribution, $20 \mathrm{~ms}$ or $40 \mathrm{~ms}$ update interval), but there was no spatial variation in dot direction within the aperture. Rather, the directions of all dots updated synchronously and identically to create a coherent, timevarying motion direction (Osborne and Lisberger, 2009). For the pursuit experiments that accompanied the physiology recordings, the target aperture had a $4^{\circ}$ diameter, with 100 dots moving with the aperture at $15-20 \%$, set according to the optimum pursuit speed for each monkey. Targets appeared $2-3^{\circ}$ eccentric to the fixation spot and immediately began translating toward it as the fixation point was extinguished. The pursuit interval lasted for $650 \mathrm{~ms}$. The MT recordings were performed while monkeys maintained fixation within $2^{\circ}$ and the motion stimulus was projected onto the unit's RF. The stimulus aperture was positioned, scaled, and motion speed was chosen to drive the unit maximally. The base direction of motion was positioned on a flank of the direction tuning $\sim 45^{\circ}$ from the neuron's preferred direction, where sensitivity to direction fluctuations is maximal.

Additional stimulus forms. We performed a limited number of experiments with alternate stimulus forms to determine to what degree the filter generalizes to other motion stimuli. We used several translating Gabor stimuli, all of which were $100 \%$ contrast, odd symmetric, 1 cycle ${ }^{\circ}$ sinusoids that translated at $15^{\circ} \mathrm{s}$. Grating orientation was always perpendicular to the direction of motion. The single Gabor stimulus had a $14^{\circ}$ diameter aperture with a $2 \mathrm{D}$ Gaussian envelope SD of $2.75^{\circ}$ and was either presented against a dark background or against a uniformly illuminated background set to the average intensity of the Gabor. We also used an array of 13 small, $2^{\circ}$ diameter Gabors $\left(S D=0.75^{\circ}\right)$ positioned $1-2^{\circ}$ apart to create an overall stimulus diameter of $14^{\circ}$ in a diamond formation. The array was presented against a background set to the mean luminance of each Gabor. We also created a 100\% contrast, $14^{\circ}$ diameter square aperture checkerboard pattern, with $1.8^{\circ}$ squares that alternated black and white, that translated together against the dark screen of the CRT. Additionally, we tested a range of fully coherent dot pattern stimuli in apertures of $5-14^{\circ}$ diameter and a dot density of $1 \mathrm{dot} / \mathrm{deg}^{2}$ that translated at $15 \%$, and a $0.5^{\circ}$ circular spot target moving at $16 \%$. The motion directions of all targets were pseudorandomly interleaved, $0^{\circ}$, $\pm 3^{\circ}$, and $\pm 6^{\circ}$ leftward and rightward.

Behavioral filter calculation. We performed a linear analysis of the correlation between fluctuations in eye direction and fluctuations in stimulus direction during the first $150 \mathrm{~ms}$ of pursuit. We chose this time period to emphasize the portion of the eye movement that is dominated by retinal image motion while the eye is still. After the target begins to move, there is a latency interval of $75 \pm 7 \mathrm{~ms}(\mathrm{SD})$ for monkeys $(n=40$ datasets; 4 subjects) and $160 \pm 11 \mathrm{~ms}(\mathrm{SD})$ for humans ( $n=9$ datasets; 3 subjects) before the eye begins to accelerate. The visually driven open loop interval is longer than the latency period, ranging from 93 to $139 \mathrm{~ms}$ in monkeys and from 236 to $320 \mathrm{~ms}$ in humans based on past experimental measurements for which the measurement criterion was known to us (Lisberger and Westbrook, 1985; Osborne et al., 2007; Mukherjee et al., 2015). The duration of the open loop interval in monkeys is often standardized to $100 \mathrm{~ms}$ when a measurement is lacking and a very conservative measure is desired. Here, our primary concern was computing the extent of the linear filter rather than restricting analysis to the open-loop interval. We chose an analysis time window of $150 \mathrm{~ms}$ as a compromise between the typical duration of the pursuit open-loop interval and adequate movement duration to characterize the motion filter fully. We used a $150 \mathrm{~ms}$ window in two ways: the time vector of pursuit considered on each trial was limited to the first $150 \mathrm{~ms}$ of pursuit and the filter at stimulus-response time lags was computed up to $150 \mathrm{~ms}$. A longer time window ensured that we could truly observe stimulus-behavioral correlations go to zero for the shortest and longest time lags. Before analysis, we shifted the eye trace with respect to the stimulus trace by $50 \mathrm{~ms}$ to compensate partially for the average pursuit latency and thereby center the filter within the $150 \mathrm{~ms}$ analysis window. Then, at each time point starting from pursuit onset, we computed the correlation between eye and the preceding dot direction values at time lags from $0 \mathrm{~ms}$ to $150 \mathrm{~ms}$ in the past (actually $50 \mathrm{~ms}$ to $200 \mathrm{~ms}$ ). As expected, these correlations were zero for the shortest and longest lags. At pursuit onset, the stimulus has not been moving for $200 \mathrm{~ms}$, so the stimulus was extended with zeros. The algorithm steps through the first $150 \mathrm{~ms}$ of pursuit computing the correlation at all lags, and then averages all samples at given time lag to determine the temporal filter. Therefore, each trial in a day's experiment contributes up to 150 measurements of the correlation at each lag value. We tested the degree to which eye movements just beyond the true openloop interval affected our results and found that the structure of the filter was stable across the first $200 \mathrm{~ms}$ of monkey pursuit, in agreement with a previous study (Osborne and Lisberger, 2009). We describe this calculation in more detail below.

The pursuit response can be described as a velocity vector $\vec{v}(t)=$ $\left(\vec{v}_{H}(t), \vec{v}_{V}(t)\right)$ where $\vec{v}$ indicates an eye velocity vector and the subscripts " $\mathrm{H}$ " and "V" label horizontal and vertical, respectively. We sampled the eye velocity at $1 \mathrm{~ms}$ intervals. To relate the eye movement to the direction perturbations in the stimuli, we converted the vector eye velocities into time-varying eye direction: $\vec{e}(t)=\tan ^{-1}\left(\vec{v}_{V}(t) / \vec{v}_{H}(t)\right)$. For each target condition, we then subtracted the trial averaged mean eye direction over time from each pursuit trial to create an array of residuals. The eye direction residual for the $i^{\text {th }}$ trial is given by the following: $\delta \overrightarrow{\mathrm{e}}_{i}(t)=$ $\vec{e}_{i}(t)-<\vec{e}_{i}(t)>$ where $\langle\ldots>$ denotes an average across trials with the same target motion. We performed a similar operation on the stimulus. First, we up-sampled the stimulus to $1 \mathrm{~ms}$ resolution, creating an array of residual dot directions over time with respect to each dot's mean direction with a target condition. We used the arrays of eye movement and stimulus residuals to compute a $3 \mathrm{D}$ linear filter $F(R, \theta$, $T$ ), which describes the spatial and temporal relationship between motion and eye movement. The filter is defined in discrete time by the following equation:

$$
\hat{e}(T)=\sum_{\tau} \sum_{\theta} \sum_{R} F(R, \theta, \tau) S(R, \theta, T-\tau)
$$

where $\hat{e}(T)$ represents the estimated instantaneous eye direction at time $T, R$ and $\theta$ are spatial coordinates of eccentricity and direction in visual space with respect to the fovea, $\tau$ is the time lag between the stimulus and the predicted response, and $S$ is the motion stimulus defined relative to the fovea (Weiner, 1949, Mulligan, 2002; Papoulis, 1991; Osborne and Lisberger, 2009; Tavassoli and Ringach, 2009). The filter is the function that minimizes the summed squared error between the estimated and actual eye direction over time. When applied to the dot motion on a single trial, the filter predicts the eye direction over time. The quality of the prediction is a measure of how linear the visual to motor transforma- 
A

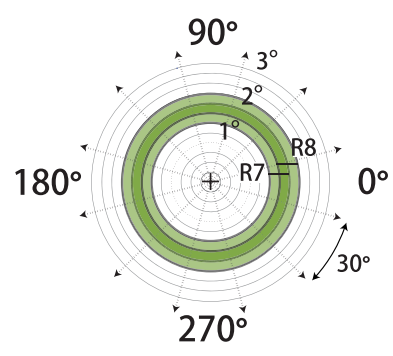

D

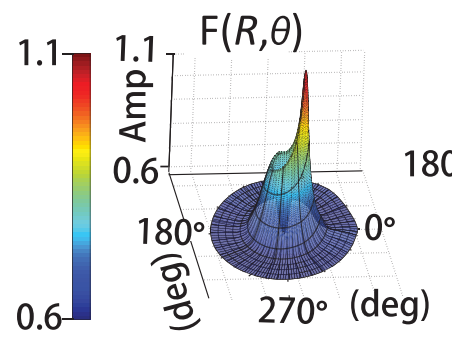

B

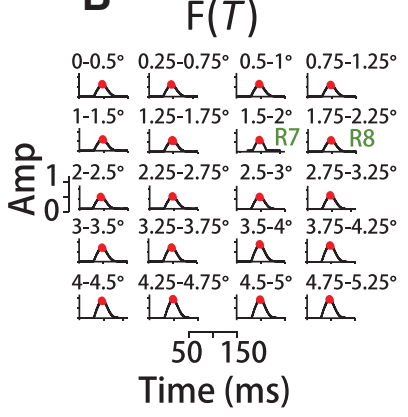

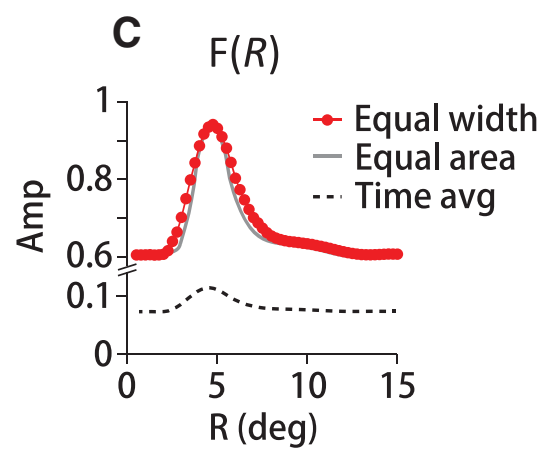

E
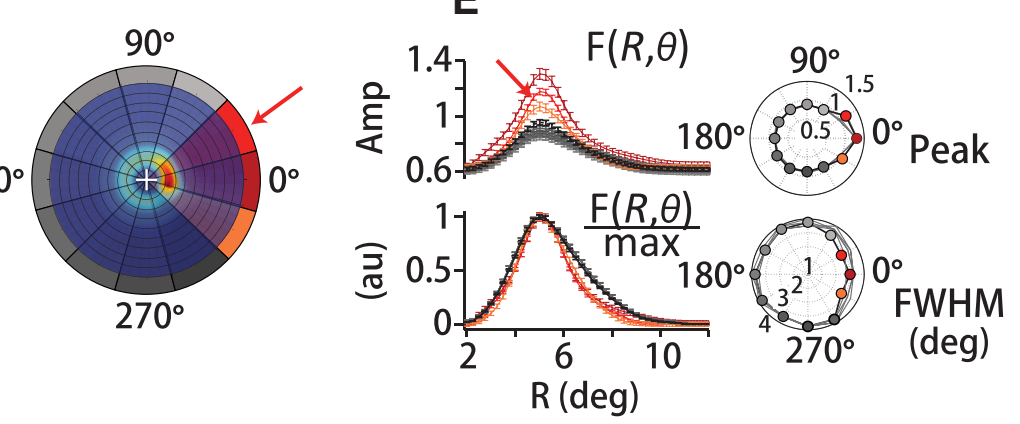

Figure 2. Spatiotemporal filter for motion integration. $\boldsymbol{A}$, We defined the motion stimulus on a polar grid centered on instantaneous eye position (+), dividing visual space into 59 concentric overlapping annuli of $0.5^{\circ}$ (visual degrees of spatial arc) width that span $15^{\circ}$ of eccentricity. Green rings (R7, R8) exemplify the overlap in adjacent annuli. We further subdivided space into $12,30^{\circ}$ pie-slice segments. $\boldsymbol{B}$, At each spatial location, we computed a temporal filter between eye and dot-averaged motion direction, here shown only for eccentricities up to $5.25^{\circ}$ of $15^{\circ}$. Red circles indicate the peak of the temporal filter, $F\left(R=R_{\mathrm{i}}, T\right)_{\max }$ Each temporal filter represents a unitless weight corresponding to a dot's contribution to eye direction based on its position and the time delay. The peak temporal filter amplitudes (Amp) sweep out the spatial filter based on motion eccentricity, $F(R)$, in $C$, red trace. Reconfiguring the annuli to equalize the number of dots in each grid region does not change the form of the spatial filter (gray trace). Defining $F(R)$ by the time-averaged amplitude rather than the peak reduces the scale, but does not change the filter's form (dotted black trace). $\boldsymbol{D}, 3 \mathrm{D}$ spatial filter, $F(R, \theta, T)$, shows a pronounced directional anisotropy, with high amplitudes in front, below and above the eye. Color and height indicate the relative contribution to pursuit of different portions of the visual field in a 3D projection (left) and a top-down view (right). The red arrow points to the same example segment (15-45 degrees of spatial arc) in $\boldsymbol{D}$ and $\boldsymbol{E}$. The gray-red colors of the polar plot's outer sections (right) represent the relative spatial filter amplitudes in each angular segment. The contribution of many segments was nearly identical, reflected in the similarity in outer-section shading. $\boldsymbol{E}$, Top left, Set of spatial filters from each directional segment from one experiment (M1), colors correspond to the right panel in $\boldsymbol{D}$. Amplitudes are high along the direction of motion (red-orange shades), but similar otherwise (gray shades). Top right polar plot, Peak amplitude versus direction segment for three humans and two monkeys. The traces overlap. Bottom left, Normalizing by the peak amplitude illustrates that the filter is narrowing in segments where amplitudes are high. Bottom right polar plot, Spatial filter FWHM for five subjects (black, gray lines).

tion is in pursuit. As defined by Equation $1, F(R, \theta, T)$ has no explicit units, but rather represents the relative weighting of motion at different spatial locations and time lags with respect to the eye direction at some point in time.

We solved Equation 1 for $F(R, \theta, T)$ using a modified version of MATLAB's tfe function (The MathWorks), which is based on Welch's averaged periodogram method. The algorithm divides the input (fluctuations in dot direction) and output (fluctuations in eye direction) into overlapping $150 \mathrm{~ms}$ sections that are detrended, padded with zeros to a length of 256 (or 512) data points, and convolved with a 256-point (or 512-point) Hann window ("Hanning") to compute the discrete Fourier transform. Most of our analyses were confined to the first $150 \mathrm{~ms}$ of pursuit. We computed the power spectral densities as the squared magnitude of the Fourier transform averaged over the overlapping sections and over trials. The algorithm normalizes the cross power spectrum between stimulus and response by the power spectrum of the stimulus. We used a cutoff frequency of $30 \mathrm{~Hz}$ for the spatiotemporal noise stimuli and a cutoff frequency of $35 \mathrm{~Hz}$ for the coherent dot motion stimuli. This operation is mathematically equivalent to walking millisecond by millisecond through the first $150 \mathrm{~ms}$ of pursuit, computing the correlation between the motion stimulus and the eye movement (and the stimulus autocorrelation) at each point at time lags from 0 to $150 \mathrm{~ms}$, and then averaging the results for each time lag and dividing the cross-correlation by the autocorrelation. The zero padding compensates for the fact that at early times in the pursuit response there were not $150 \mathrm{~ms}$ of prior stimulus.

There was not a sufficiently large data sample to measure the filter at the pixel resolution of our display. Instead, we divided visual space into a polar grid centered on the fovea at each time step. We divided the visual field eccentrically into 59 overlapping concentric annuli ( 0 to 0.5 visual degrees of spatial arc, $0.25^{\circ}$ to $0.75^{\circ}, 0.5^{\circ}$ to $1^{\circ}, \ldots, 14.5^{\circ}$ to $15^{\circ}$ ) and angularly into $12,30^{\circ}$ pie slice segments $\left(-15^{\circ}\right.$ to $+15^{\circ},+15^{\circ}$ to $+45^{\circ}, \ldots$ ) (see Fig. $2 A$ ). Annuli of increasing eccentricity encompass a greater area of the visual field, and hence a larger number of dots. To ensure that the filter structure was not affected by the spatial binning, we normalized by number of dots for each location and time step in the calculation (Fig. $2 C$, red trace). As a control, we also confirmed that segmenting visual space such that equal numbers of dots fell within each area produced an identical spatiotemporal filter (Fig. $2 C$, gray trace). To analyze the filter's directional structure, we flipped the sign of the horizontal component of target and eye velocity on leftward trials. This operation oriented the angular coordinate frame with respect to the direction of motion set to $0^{\circ}$ while preserving upward and downward motion components across all stimulus directions. We then computed $F\left(R, \theta=\theta_{\mathrm{i}}, T\right)$ separately for each section $i$ (Fig. $\left.2 D, E\right)$.

We used a cross-validation resampling technique, measuring the filter with a random draw of $70 \%$ of the sample and then using the filter to predict the eye's response to the remaining $30 \%$ of the stimuli. We repeated this process many times to generate a mean and error (SD) for all measurements. We created an array of residuals, or prediction errors, by subtracting the predicted eye direction over time from the actual eye movement. We inspected the residual differences between the filterpredicted and measured eye movements for evidence of response saturation or other static (time independent) nonlinearities. To quantify the predictive power of the linear filter, we calculated the correlation coeffi- 
cient between the predicted and actual eye movements over time as part of the cross-validation analysis.

Analysis of spatial anisotropy and dynamics in the motion filter. To determine whether there are any rotational anisotropies in motion integration, we analyzed pursuit data as a function of direction with $30^{\circ}$ diameter aperture noisy dots stimuli. As described above, we divided the visual field into 12 sections, each subtending 30 visual degrees of spatial arc. We centered the analysis frame on the eye's position at each time step. We flipped the sign of the horizontal components of the stimulus and eye velocity on leftward trials to keep the coordinate frame consistent across all motion directions. The filter calculation proceeded as described above. To determine how the form of the spatial and temporal filter components changed over time, we performed the analysis in overlapping $150 \mathrm{~ms}$ windows that spanned the first $450 \mathrm{~ms}$ of the pursuit response.

Comparison of pursuit and perception. Because we could not compute the spatiotemporal filter directly from our 2AFC perceptual data, we instead quantified how well the pursuit filter predicted subjects' perceptual choices compared with other filter shapes. We analyzed data from $0^{\circ}$ or $180^{\circ}$ trials only from experiments with $30^{\circ}$ diameter stimuli. First, we fit each subject's temporal filter with a Gaussian function, $f(x)=a_{1} e^{-\left(x-b_{1} / c_{1}\right)^{2}}$ and each spatial filter was fit with the sum of two normal functions to capture the skew, $f(x)=a_{1} e^{-\left(x-b_{1} / c_{1}\right)^{2}}+a_{2} e^{-\left(x-b_{2} / c_{2}\right)^{2}}$. All fits had $R^{2}$ values of 0.99 . Subject H1's temporal filter fit parameters were $a_{1}=0.61, b_{1}=48, c_{1}=13$, The spatial filter fit parameters were $a_{1}=0.31, b_{1}=4.8, c_{1}=1.7, a_{2}=0.64$, $b_{2}=8.2, c_{2}=28$. Subject H2's temporal filter had fit parameters $a_{1}=0.61, b_{1}=43, c_{1}=13$ and spatial filter fit parameters $a_{1}=0.33$, $b_{1}=4.5, c_{1}=1.8, a_{2}=0.64, b_{2}=9.5, c_{2}=24$. Then, a set of $209(19 \times$ 11) spatial and $208(16 \times 13)$ temporal filter forms was generated by changing the peak eccentricity or delay $\left(b_{1}, b_{2}\right)$ and the width (related to $c_{1}, c_{2}$ ) systematically. We tested different spatial filter forms while keeping the temporal filter constant and vice versa. Nine examples from the spatial filter set are illustrated in the margins of Figure $5 \mathrm{~A}$ by colored dashed lines holding the other fit parameters constant.

To evaluate the predictive performance of each filter form, we convolved the spatiotemporal filters, $F_{\text {model }}(R) F_{\text {model }}(T)^{\mathrm{T}}$, with the dot motion on each trial to predict the pursuit or perceptual response and then we computed the linear correlation between the predicted responses and the actual responses over all trials. We assigned perceptual responses +1 for upward and -1 for downward choices. For the pursuit data, we computed the mean eye direction in a $5 \mathrm{~ms}$ window centered at $250 \mathrm{~ms}$ after motion onset and assigned the trial +1 if the eye direction was rotated upward and -1 if it was rotated downward with respect to the mean. For the perception data, we used each filter form to predict the time-averaged motion direction over the $250 \mathrm{~ms}$ interval, as per the task we assigned the subjects. Then, we computed the correlation coefficient between the binary behavioral data (eye direction or perceptual report) and the predicted responses from the filters in degrees. The correlation calculation was therefore identical for the pursuit and perceptual data. We converted the correlation value for each filter shape into a $z$-score by subtracting the mean value of the correlation coefficient across all model filter forms and dividing by the SD of the values across the set. The $z$-score transformation simplified comparison between pursuit and perception predictions. To quantify how similar the pursuit data and perception data matrices were, we formed distributions of $z$-scored correlation coefficients for all model filters and compared the RMS variance of those distributions for each subject. We performed a control analysis to ensure that the pattern of behavioral predictions by the different spatial and temporal filter forms did not arise by chance. We took multiple random draws of $50 \%$ of the pursuit or perception data samples, convolving each of the 208 or 209 filter shapes with the stimulus set, to predict behavioral responses. We then computed the correlation coefficient between the predicted and actual behavioral responses and the correlation coefficient between the predicted responses and a random permutation of the responses. We defined the $p$-value of each filter form as the fraction of times that the correlation with the randomly permuted values equaled or exceeded the actual correlation coefficient. The $p$-values were $\leq 0.01$ for all correlation coefficients plotted in Figure 5.
We performed a second control analysis to ensure that differences in how we used each filter form to predict eye directions or perceptual reports did not affect the results. To generate the results in Figure 5, we used the filter sets differently to predict the perceptual and pursuit responses based on the difference between the tasks. In the perception tasks, subjects reported whether the time-averaged motion direction was upward or downward from horizontal, so we used each model filter form to predict the time-averaged dot direction across the $250 \mathrm{~ms}$ motion interval. In pursuit, the eye reports the estimated direction at each time point, so we used each filter to predict the eye direction at $250 \mathrm{~ms}$ after pursuit onset. To ensure that the data-handling difference did not contribute to the grayscale patterns of correlation values in Figures 5, $A$ and $B$, we reanalyzed the data to test both prediction methods on each dataset. We generated matrices like the right panels in Figures $5 A$, but we used the temporal filter set to estimate the time-averaged pursuit stimulus direction over $250 \mathrm{~ms}$. The pursuit prediction results were not statistically different from the data in Figure $5 A$ ( $p=0.41$, two-sample $t$ test, $\mathrm{H} 1 ; p=0.37, \mathrm{H} 3)$. Next, we used the temporal filter set to predict the perceptual stimulus direction at $250 \mathrm{~ms}$ to correlate with the perceptual reports. Again, the results were not statistically different from the right panels in Figure $5 B(p=0.43$, two-sample $t$ test, $\mathrm{H} 1 ; p=0.39, \mathrm{H} 3)$. Therefore, the method used to characterize the stimulus for pursuit and perception data did not affect the results reported in Figure 5.

Neural temporal filter calculation. We computed the temporal filter relating fluctuations in spike count to stimulus direction as a function of time lag using the same methods described above for the behavioral filters. We counted spikes in overlapping $20 \mathrm{~ms}$ time windows centered at $1 \mathrm{~ms}$ intervals starting from stimulus motion onset and extending over a 250-2000 ms motion segment for each trial. The calculation of the neural filter then proceeds identically to the methods described for the behavioral filter using a cutoff frequency of $30 \mathrm{~Hz}$ and replacing eye direction with spike count in the time window. We computed the correlation between the count values and motion direction at $1 \mathrm{~ms}$ intervals up to lags of $200 \mathrm{~ms}$.

Separability analysis. We performed singular value decomposition to determine the extent to which motion filters were space-time separable; that is, the degree to which the full spatial temporal filter is captured by the outer product of a 2D spatial and 1D temporal filter, $F(R, \theta) \otimes F(T)$. We analyzed both $F(R, T)$ (59 eccentricity annuli by 150 time points) and the full 3D filter, $F(R, \theta, T)$, after reorganizing into a sparse, $2 \mathrm{D}$ array of 708 spatial points (12 direction segments $\times 59$ annuli) by 150 time points. The relative squared weight of the first singular value $\sigma_{1}$ with respect to the others; that is, $\sigma_{1}^{2} / \Sigma_{i} \sigma_{i}^{2}$ defines the separability index (SI). An SI of 1 indicates that variance in the filter form is perfectly captured by a single mode and thus its space-time separability is exact.

Optic flow score. To determine whether the filter computed with noisy dots stimuli also predicts responses to novel stimuli, we compared the filter predictions with measured eye movements. We convolved the filter with each new stimulus and then integrated over space and time from motion onset to $150 \mathrm{~ms}$ to generate a scalar value termed the optic flow score. The optic flow score summarizes the net motion energy in a stimulus as weighted by the visual system (Mukherjee et al., 2015). To perform the convolution, we first converted each new motion stimulus into a pixel flow field using an algorithm based on Sun et al., 2010. The pixel flow field is a luminance-weighted vector field describing the translation of each pixel from one frame to the next. For dot pattern stimuli, the pixels were either on (1) or off (0), so the optic flow for adjacent frames was simply the fraction of illuminated pixels per frame times their speed along the base direction of motion summed over dots and frames. The optic flow for a stimulus with intermediate luminance values such as a Gabor is determined by the speed-weighted difference in pixel intensity with respect to the background for each pair of frames normalized by the total pixel number. For non-zero background illumination, darker pixels within a stimulus such as a grating create negative flow values with respect to the background. However, the same grating stimulus against a dark background will have non-negative flow scores. For all frames in the first $150 \mathrm{~ms}$ of motion, we measured the position of illuminated pixels with respect to eye position and applied the MATLAB function conv2 to compute the $2 \mathrm{D}$ convolution between the filter $F(R, \theta, T)$ and the 
luminance-weighted pixel flow for each pair of frames and at each spatial grid location. The convolution operation produces a scalar flow score for each stimulus form that we compared to the measured peak eye acceleration. The scores that we report are averages across all trials in a dataset $(n \geq 200)$. The stimuli for which we computed flow scores matched the sizes of the noisy dots stimuli that generated the set of filters, so no interpolation of the filter form was necessary except for a $2^{\circ}$ aperture dot stimulus and a $0.5^{\circ}$ spot target. We generated a flow score for a $2^{\circ}$ aperture stimulus by extrapolating the foveal eccentricity of the spatial filter peak based on the observed linear relationship between the location of the spatial filter peak and stimulus radius. The spot target was so small compared with the stimuli for which we computed filters that we had to estimate the score differently. We used two scores based on different assumptions: one without any filtering (i.e., the number of illuminated pixels $\left(180 \times 16^{\circ} / \mathrm{s} \times 150\right.$ frames $\left.=4.32 \times 10^{5} \% \mathrm{~s}\right)$ and another multiplying the unfiltered score by a factor equal to the extrapolated estimate of filter amplitude for a $0.5^{\circ}$ stimulus $\left(4.32 \times 10^{5} \% \mathrm{~s} \times 0.4=17.3 \times 10^{4}\right.$ $\%$ ) using the filter peak location versus size relationship (defined above) that we derived based on larger stimuli.

\section{Results}

To determine how visual motion signals are integrated across space and over time, we performed experiments to measure pursuit eye movements in response to stochastic motion stimuli composed of random dot kinematograms. We base our approach on the "noisy dots" stimuli developed by Williams et al. to study global motion integration (Heinen and Watamaniuk, 1998; Watamaniuk and Heinen, 1999; Osborne and Lisberger, 2009; Watamaniuk et al., 2011; Mukherjee et al., 2015) (Fig. 1A). To drive pursuit effectively, each dot in the stimulus pattern has a constant drift direction and speed, but with an added stochastic direction perturbation that we updated every $40 \mathrm{~ms}$ (4 frames) (Fig. $1 B$; see Materials and Methods). The stochastic component of each dot's motion generates a random walk around the constant motion vector. Dot perturbations update independently but synchronously, which differentiates the stimulus direction across space and time. The overall stimulus appearance is of a swarm that translates together, but with each member executing its own randomized flight path. The spatial and temporal variation in the stimulus allows us to correlate eye direction with stimulus direction as a function of location in the visual field and time delay to compute the spatiotemporal motion filter. With the exception of the stochastic element of dot motion, our experiments were otherwise standard pursuit tasks (Materials and Methods). We focus our analysis on the first $150 \mathrm{~ms}$ of pursuit that is driven by visual motion estimates without a substantial contribution from extraretinal feedback (Lisberger and Westbrook, 1985; Mukherjee et al., 2015).

\section{Spatiotemporal filter for motion direction}

From the eye and target direction over time, we used linear analysis to compute the spatiotemporal filter for motion integration within this experimental context (see Materials and Methods). We sampled the motion stimulus and the eye velocity at $1 \mathrm{~ms}$ intervals. We also divided visual space into overlapping $0.5^{\circ}$-wide annuli. Each annulus was centered on the position of the fovea and then subdivided into 12 segments spanning 30 visual degrees of spatial arc (see Materials and Methods; Fig. 2A). We then stepped through the eye movement time vector on each trial and computed the linear correlation between fluctuations in eye direction and the vector-average of the dot directions at each spatial location in the visual field $(R, \theta)$ as a function of time delay, $T$, from 0 to $150 \mathrm{~ms}$ in the past in $1 \mathrm{~ms}$ increments. At each step in the calculation, we normalized by the autocorrelation of the mo- tion stimulus (see Materials and Methods). This calculation solves Equation 1 to find $F(R, \theta, T)$, a unitless function of space and time that best predicts the eye direction at each time point from past stimulus motion in each region of the visual field. Although dot density is uniform within the stimulus aperture on average, the number of dots falling within each spatial region at each time point and trial is not identical. Throughout the analysis, we correct for varying dot number by normalizing by the number of dots falling within each spatial region at each time step. Therefore, the filter that we derive represents the contribution that each dot will make to the eye movement based on its spatial location over time.

The filter that we derive captures the relationship between fluctuations in stimulus direction and fluctuations in eye direction, our proxy for visual motion estimates. We present the motion filter in several ways. The 20 panels in Figure $2 B$ are a set of temporal filters, $F\left(R=R_{\mathrm{i}}, T\right)$, from a monkey pursuit experiment with a $30^{\circ}$-diameter noisy dot stimulus. The filters were computed on a grid of concentric annuli (Fig. 2A) at eccentricities of $R_{\mathrm{i}}=0-14.75^{\circ}$, although only the first 20 bands $R_{1-20}=0-4.75^{\circ}$ are shown. The overlap between annuli is illustrated by the greenshaded bands in Figure $2 A$. To generate the temporal filters in Figure $2 B$, we stepped through time points and trials, computing the correlation between eye direction and vector mean dot direction within each annulus as a function of time lag (see Materials and Methods). The red dots mark the peak amplitude at each eccentricity and collectively trace out the rotationally averaged spatial filter, $F(R)$ (red trace; Fig. 2C). If we represent $F(R)$ as the time-averaged amplitude instead of the peak value of each temporal filter in Figure $2 B$, we change the scale, but not the form of the spatial filter (black dashed line; Fig. 2C). The most striking feature of the spatial filter is that its amplitude peaks eccentric to the fovea, at $\sim 5^{\circ}$ (visual degrees of spatial arc) for the $30^{\circ}$ diameter stimulus in this experiment. Contributions from motion at eccentricities $>10^{\circ}$ and $<2.5^{\circ}$ with respect to gaze position are only $\sim 60 \%$ of the peak. Using alternate spatial partitions that equalized the number of illuminated pixels (i.e., dots) within each segment produced a nearly identical spatial filter (gray trace; Fig. 2C; see Materials and Methods). The preferential weighting of eccentric motion was consistent across the range of stimuli that we tested.

The full spatial filter, $F(R, \theta)$, shows a pronounced directional anisotropy. In Figure $2 D$, both color and height indicate the relative contribution of motion signals at different spatial locations within the visual field to global direction estimates. The coordinate frame is centered on the eye's position, and $0^{\circ}$ corresponds to spatial locations along the axis of stimulus motion. The data are drawn from the same example experiment as the other panels. To calculate the rotational component of the spatial filter, we divided visual space into 12 pie-slice segments of 30 degrees of spatial arc (i.e., $-15^{\circ}$ to $+15^{\circ},+15^{\circ}$ to $+45^{\circ}$, etc.; see Fig. $2 D$, right) and computed the spatial filter within each slice separately using the same eccentricity annuli described above. We oriented the coordinate frame on the target direction so that the $0^{\circ}$ segment always represented locations "ahead" of the eye along the direction of stimulus motion. We used both leftward and rightward trials, so we flipped the sign of the horizontal components of leftward motion to align with the same coordinate frame. Therefore, the vertical segments (labeled $90^{\circ}$ and $270^{\circ}$ ) always represent spatial locations above and below the eye, respectively. We found substantial direction-dependent anisotropy in the spatial filter (Figs. 2D,E). Figure 2D, left, presents the spatial filter $F(R, \theta)$ as a pseudo-3D projection and the right panel the same 
A

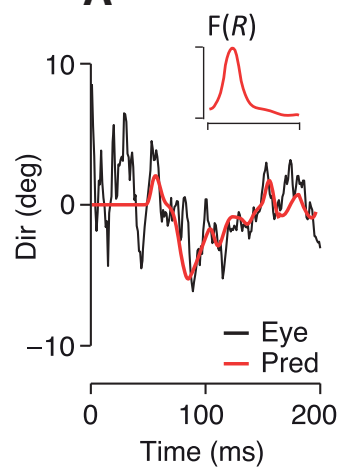

B

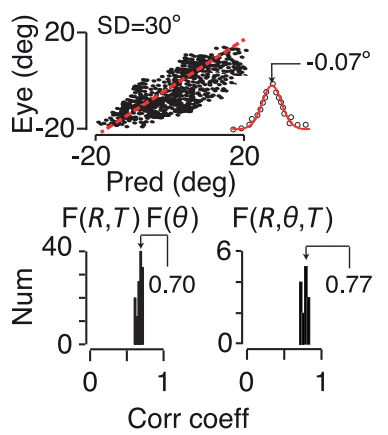

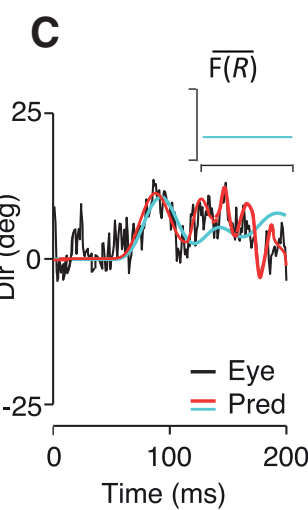

D

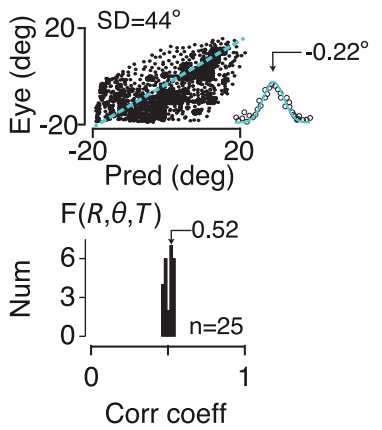

Figure 3. Predictive power of the spatiotemporal filter for pursuit. $\boldsymbol{A}$, Predicted eye direction over time with respect to pursuit onset for an example trial (red) compared with the monkeys' actual eye direction (black). The inset shows the eccentricity dependence of the spatial filter, but the full 3D filter, $F(R, \theta, T)$ was used for the reconstruction (black trace). $\boldsymbol{B}$, Although we calculated the filter by relating fluctuations in stimulus direction with eye direction around the mean, we found that the same filter form is able to predict the time averaged eye direction throughout the dataset (top) The predictive errors have a Gaussian distribution and near-zero bias (inset) indicative of a noise process. The dashed red line has a slope of 1 . Bottom, Population data showing the distribution of correlation coefficients between predicted and actual eye direction over time across many datasets and subjects. The bottom left panel shows the correlation coefficients using $F(R, T) \otimes F(\theta)$ for experiments with $5-30^{\circ}$-diameter stimuli. The right panel shows just the $30^{\circ}$-diameter experiments from which we derived $F(R, \theta, T)$. $\boldsymbol{C}$, Eye direction prediction is poor with a flat spatial filter, $\overline{F(R)}$ (cyan line, inset) despite pooling motion over all dots to estimate the mean direction. Predicted (cyan) and measured eye direction (black) across time for an example trial are shown with the actual pursuit filter's prediction in red. $\boldsymbol{D}$, Top, Predicted versus actual eye direction as in $\boldsymbol{B}$. The distribution of residuals shows that the filter produces both systematic and random prediction errors. Bottom, as in $\boldsymbol{B}$. The flat filter accounts for only $27 \%$ of the variance of eye direction over time compared with $59 \%$ for the filter itself.

data as if viewed from above. The colors (orange to red, grays) of the outer borders of the direction segments around the right panel of Figure $2 D$ identify the region of the visual field from which the filter traces in Figure $2 E$ were derived. For example, a red arrow points to the $+15^{\circ}$ to $+45^{\circ}$ spatial segment in Figure $2 D$ and to the corresponding plot of $F\left(R, \theta=\theta_{i}\right)$ in Figure $2 E$. The filter amplitudes are higher in the spatial segments centered from $-30^{\circ}$ to $+30^{\circ}$ (Fig. $2 \mathrm{D}, \mathrm{E}$, orange-red colors, right panel) compared with all other segments (Fig. 2D,E, gray colors). Filter amplitudes are so similar in all other direction segments that the grayscales are difficult to differentiate. The largest contribution to pursuit is from the $0^{\circ}$ segment, representing motion in front of the eye (Fig. 2E, top left panel, and top polar plot). We find the same directional pattern whether we analyze the stimulus motion or the retinal image motion, the difference between eye and target velocity. The directional enhancement has a very similar pattern of spatial weighting for all human (H1-H3) and monkey (M1M2) subjects, such that the traces in Figure 2E's polar plots are difficult to resolve. The directional enhancement is not solely a multiplicative rescaling because the filters from each direction segment do not superimpose after normalization, $F(R, \theta)$ / $\max (F(R, \theta))$. The motion filters in spatial direction segments in front of the eye are narrower as well as higher in amplitude. We present the normalized data for all five subjects in the bottom left panel and bottom polar plot in Figure 2E. Before normalization, the filter's full width, measured at half maximum (FWHM), is slightly narrower in the same segments in which amplitudes are enhanced (Fig. 2E, red colors, bottom panel). The filter width in the spatial segment in front of the eye $\left(0^{\circ}\right)$ was $27 \pm 4 \%(\mathrm{SD})$ smaller than the width of the segment behind the eye $\left(180^{\circ}\right)(n=12$ datasets, $\mathrm{H} 1-\mathrm{H} 3, \mathrm{M} 1-\mathrm{M} 2)$. The narrowing of the leading versus trailing direction spatial filters was statistically significant across subjects $\left(p<3 \times 10^{-4}, n=\right.$ 12 datasets, paired $t$ test). The difference in normalized filter amplitudes was somewhat smaller, $18 \pm 3 \%$ (SD), but still statistically different from zero $\left(p=3 \times 10^{-4} ; n=12\right.$ datasets, paired $t$ test). The directional anisotropy that we observed suggests both a gain enhancement and a slight spatial sharpening for motion integration in and near the direction of motion.
We did not find a corresponding directional difference in the temporal filters that would indicate that motion integration time scales differed by spatial direction. We performed a paired $t$ test on five subjects (H1-H3, M1-M2) comparing temporal filters from the $0^{\circ}$ and $180^{\circ}$ direction segments and found that there were no significant differences in the temporal filter peak time delay ( $p=0.65, n=12$ datasets) or duration (FWHM) $(p=0.74$, $n=12$ datasets).

\section{Predictive power for pursuit}

The narrow peak of the spatial filter is surprising because it indicates that the visual system is down weighting motion information from a large part of the visual field. That seems counterintuitive because all of the dots in the pattern are equally informative about the global motion direction. However, we found that the spatial filter predicts the eye direction over time quite well, whereas an "ideal observer" spatial filter that sums motion uniformly over the visual field does not (Figs. $3 A, C$, top insets). We tested filter performance by applying the filter to trial data left out of the filter calculation to predict the eye direction over time. The prediction for an example trial with a $30^{\circ}$-diameter noisy dots stimulus using the $3 \mathrm{D}$ pursuit filter, $F(R, \theta$, $T$ ), is shown in Figure $3 A$ (red trace) and is quite similar to the actual eye direction over time (black trace). The relationship between the predicted and actual eye direction at many time points throughout the dataset is plotted in Figure $3 B$ (top). Although we calculated the filter by relating fluctuations in stimulus direction to fluctuations in eye direction around the mean, we found that the same filter form also predicts the time-averaged eye direction correctly (Fig. 3B, top). Therefore, the spatiotemporal filter in Figure 2 captures global direction estimates for pursuit, not just the eye's response to small direction fluctuations. The prediction errors have a Gaussian distribution and a near-zero bias of $0.07^{\circ}$ (Fig. $3 B$, inset, top), indicating that the filter accounts for the structured variation in motion estimation leaving only residual noise. We computed the linear correlation coefficient, $\rho$, between the predicted and actual eye direction over the first 150 ms of pursuit to summarize the quality of the filter's prediction for each dataset (Fig. 3B, bottom). We found that the full 3D spatiotemporal filter, $F(R, \theta, T)$, accounts for almost $60 \%$ of the variance $\left(\rho^{2}\right)$ in eye direction (Fig. $3 B$, bottom right) in monkeys 
$(\rho=0.77 \pm 0.4 \mathrm{SD}, n=10$ datasets, M1M2) and $62 \%$ in humans $(\rho=0.79 \pm 0.3$ $\mathrm{SD}, n=5$ datasets, $\mathrm{H} 1-\mathrm{H} 3)$. If we include only open-loop pursuit (125 ms) the fraction of variance predicted was slightly lower: $55 \%$ in monkeys ( $\rho=0.74 \pm 0.3 \mathrm{SD}, n=10$ datasets, M1-M2) and 56\% in humans ( $\rho=$ $0.75 \pm 0.3 \mathrm{SD}, n=5$ datasets, $\mathrm{H} 1-\mathrm{H} 3)$. We had sufficient dot numbers to generate the full $3 \mathrm{D}$ filter only for $30^{\circ}$-diameter noisy dots stimuli, but we found that the direction weighting, $F(\theta)$, derived from the large stimuli improved filter predictions across the full range of stimulus sizes we tested. The directionally averaged $2 \mathrm{D}$ filter, $F(R, T)$, accounts for $46 \%$ of the variance $(\rho=0.68 \pm$ $0.3 \mathrm{SD}, n=120$ datasets, M1-M2, H1-H3), whereas using the directional weighting with the $2 \mathrm{D}$ filter, $F(R, T) \otimes F(\theta)$, makes slightly better predictions of eye direction over time across all stimulus sizes (49\%, $\rho=0.70 \pm 0.2 \mathrm{SD}, n=120$ datasets, M1-M2, H1-H3) (Fig. 3B, bottom left). The difference is small but statistically significant across all subjects and stimulus sizes $\left(p=2.1 \times 10^{-11}, n=120\right.$, paired $t$ test $)$.

How well does motion across all parts of the visual field predict pursuit? Because the random walk of each dot is drawn from the same distribution, all dots are equally informative about the average direction of motion. An ideal motion estimator would therefore average over all dots uniformly to determine the global motion direction. The nonuniformity of the spatial filter suggests that the brain does not act as an ideal observer and that predictions of eye direction based on the ideal observer model should be poorer than the filter shown in Figure $3 A$ (inset). We tested the predictive power of a flat eccentricity filter, $\overline{F(R)}$, scaled to the mean spatial filter amplitude (cyan line, inset; Fig. $3 C$ ) with the same example dataset shown in Figures 2 and $3 A$. We preserved the direction weighting plotted in Figure $2 E$ to isolate the eccentricity filter's effect on prediction quality. The flat filter model can be written as follows: $(\overline{F(R)} \otimes F(\theta)) \otimes F(T)$ where $\otimes$ indicates an outer product. We found that the flat filter (cyan trace) did not predict the eye direction over time as well as the actual pursuit filter (Fig. 3C, red trace). The distribution of residuals suggests that the flat filter produces both systematic and random prediction errors (Fig. 3D, top). Across datasets, the flat filter accounts for only $27 \%$ of the variance of eye direction over time ( $\rho=0.52 \pm$ $0.3 \mathrm{SD}, n=25$ datasets, all subjects; $\rho=0.50 \pm 0.3 \mathrm{SD}, n=18$ datasets, $\mathrm{M} 1-\mathrm{M} 2 ; \rho=0.52 \pm 0.2, n=7$ datasets, $\mathrm{H} 1-\mathrm{H} 3)$. Although a flat spatial filter pools motion equally over all dots, as would an ideal observer, its predictive power is poorer than the spatially anisotropic filter that we measured from pursuit. The visual system's emphasis on a portion of the visual field is curious because it means that all available information is not being used to estimate the global motion direction to initiate pursuit. To determine whether the filter that we measured from pursuit indeed arises from internal motion estimates rather than a pursuit-specific process, we compared the motion filters in human and monkey subjects and then tested the filter's ability to predict perceptual direction estimates.

\section{Comparison between humans and monkeys}

We find that human and monkey pursuit filters are quite similar, although there is an overall time shift corresponding to the difference in pursuit latencies. For $30^{\circ}$-diameter stimuli, the peak eccentricity of the spatial filter, $F(R)$, falls at $R_{\text {peak }}=4.4 \pm 0.12^{\circ}$ (SD) for humans ( $n=4$ datasets, $\mathrm{H} 1-\mathrm{H} 3)$ and $R_{\text {peak }}=4.8 \pm 0.1^{\circ}$ (SD) for monkeys ( $n=8$ datasets, M1-M2) (Fig. $4 A$, top left). The dotted lines in Figure $4 A$ are meant only to emphasize the species differences and similarities in the filter parameters. The peak eccentricity of monkey and human spatial motion filters are close and that is also true if we subdivide space into 12 directional segments to compute $F(R, \theta)$ (see Fig. 2): $R_{\text {peak }}=$ $4.8 \pm 0.2^{\circ}(\mathrm{SD})$ for monkeys $(n=96,12$ direction segments, 8 datasets, M1-M2, $p=0.54$, one-way ANOVA), $R_{\text {peak }}=4.5 \pm$ $0.2^{\circ}(\mathrm{SD})$ for humans $(n=48,12$ directions, 4 datasets, $\mathrm{H} 1-\mathrm{H} 3$, $p=0.77$, one-way ANOVA). We characterize the width of the spatial filters by the FWHM. FWHM values in visual degrees of spatial arc are $3.2 \pm 0.1^{\circ}(\mathrm{SD})$ for monkeys $(n=8$ datasets, M1-M2) and $3.1 \pm 0.2^{\circ}$ (SD) for humans ( $n=4$ datasets, H1$\mathrm{H} 3$ ) (Fig. $4 A$, left). There is a more marked species difference in the temporal filters. Monkeys' temporal filters peak at shorter delays compared with humans (solid vs dotted lines; Fig. 4A, top right; Fig. $4 B$, bottom). The delay at which the temporal filter peaks is a measure of the time lag of the eye's response to fluctuations in motion direction. Considering just the $30^{\circ}$ stimuli, monkeys' temporal filters peaked at $T_{\text {peak }}=95 \pm 4 \mathrm{~ms}(\mathrm{SD})(n=$ 8 datasets, M1-M2) compared with $T_{\text {peak }}=185 \pm 7 \mathrm{~ms}$ (SD) for humans ( $n=4$ datasets, H1-H3) (Fig. $4 A$, top right), but in fact the temporal filters were very similar across all stimulus sizes, $T_{\text {peak }}=92 \pm 6 \mathrm{~ms}(\mathrm{SD})(n=114$ datasets, M1-M2) for monkeys. Although pursuit latencies tend to be somewhat longer than visual response delays while the eye is in flight (Krauzlis and Lisberger, 1994), the species difference in temporal filter delays is consistent with the species difference in pursuit latencies. Average pursuit latencies in monkeys with dot motion stimuli are $75 \pm 7 \mathrm{~ms}(\mathrm{SD})(n=40$ datasets, M1-M2) and in humans are $160 \pm 13 \mathrm{~ms}(\mathrm{SD})$ ( $n=15$ datasets, H1-H3), a difference of $85 \mathrm{~ms}$ on average, which is close to the $90 \mathrm{~ms}$ difference in peak delays. Because visual perception in macaques and humans is quite similar (Orban et al., 2004), the differences in temporal filters likely arise from species differences in motor processing or eye movement execution.

The duration of the temporal filters is more similar in monkeys and humans and consistent across all stimuli. The duration or width of the temporal filter describes the time window over which past motion is summed to estimate target direction and drive pursuit-the sensory-motor integration time. Pursuit filter 
A

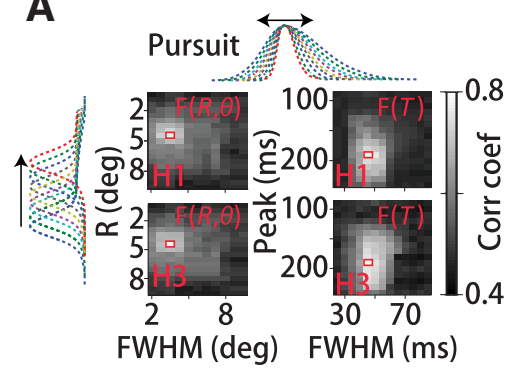

B
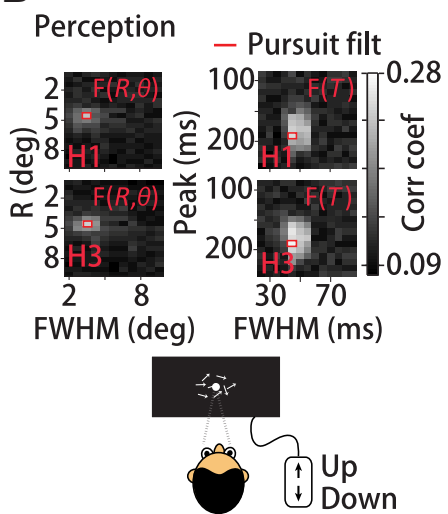

C

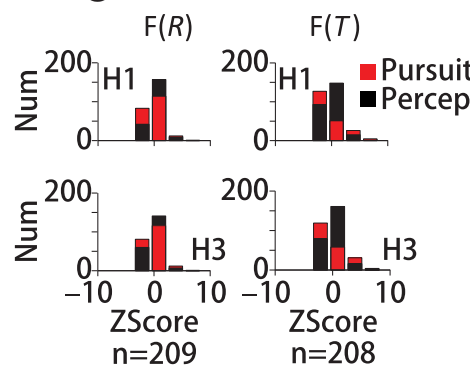

Figure 5. Same spatiotemporal filter predicts pursuit and perceptual reports of motion direction. We compared the predictive power of the actual spatial and temporal filters (red rectangles) and synthetic filters created by shifting ( $y$-axes) and stretching ( $x$-axes) the measured filter forms (top, side dotted lines, $\boldsymbol{A}$ ). Each grayscale element in the density plots represents the linear correlation coefficient between predicted and measured eye direction or perceptual reports using a different filter shape; white indicates high and black low correlation. The elements outlined in red correspond to the best prediction, obtained in each case by the actual pursuit filter. $\boldsymbol{A}$, Pursuit data for two human subjects (H1, H3). Data in left panels were generated by holding the temporal filter constant and manipulating the spatial filter; for right panels, the opposite was done. The pattern of near white values indicates the sensitivity of prediction quality to the shape of the filter. $\boldsymbol{B}$, Perceptual data for the same subjects using the same filter set. As with pursuit, the actual spatial and temporal filters yield the best prediction of perceptual reports. $\boldsymbol{C}$, Histogram plots of the z-scored correlation coefficients for pursuit and perception for manipulations in the spatial filter form (left) or temporal filter forms (right) from the same data.

A

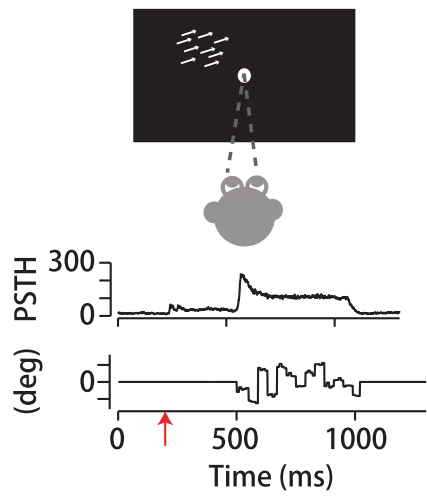

B
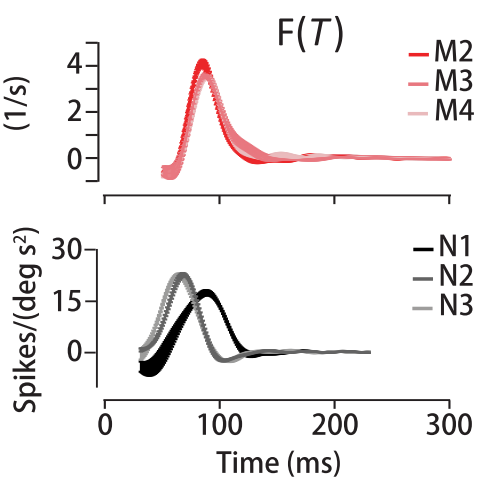

C
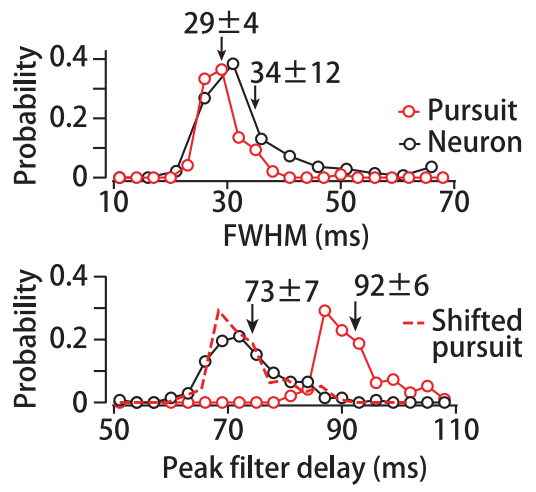

Figure 6. Temporal motion integration in MT neurons. $\boldsymbol{A}$, Top, Neurophysiology experimental design. Monkeys maintained fixation while a dot stimulus moved in the neuron's RF. Middle, PSTH of a single neuron Bottom, Stimulus direction over time. Red arrow represents stimulus appearance. Dots appeared and remained stationary for 200 ms to allow visual onset responses to decay. $\boldsymbol{B}$, Temporal filters computed from pursuit in three monkeys (top) and three MT neurons from the same monkeys (bottom). C, Top, Population distribution of temporal filter durations, FWHM, of MT neurons (black) and pursuit (red) from the same monkeys. Bottom, Distribution of temporal filter peaks for MT neurons (black solid line) and pursuit (red solid line). Matching the mean delay of the pursuit distribution to the neural data (dashed red line) shows that the distributions overlap.

durations average $42 \pm 4 \mathrm{~ms}(\mathrm{SD})$ for humans $(n=4$ datasets, $\mathrm{H} 1-\mathrm{H} 3)$ and $28 \pm 5 \mathrm{~ms}$ (SD) for monkeys ( $n=8$ datasets, M1M2) with $30^{\circ}$ diameter stimuli (Fig. $4 A$, bottom right) and do not differ with stimulus size (FWHM $=27 \pm 5 \mathrm{~ms}$ (SD), $n=114$ datasets, M1-M2, $p>0.9$, two-sided Wilcoxon rank-sum test). The filter durations for the noisy dot stimuli are comparable to those measured for coherent motion stimuli (Osborne and Lisberger, 2009).

\section{Motion filter predicts perception}

Is the spatiotemporal pursuit filter dominated by visual or motor processing? Smooth eye movements, especially during the initial open-loop interval, are guided by visual cortical inputs but generated by downstream motor circuits. Therefore, both sensory integration and motor processing may shape the filter forms we measure from eye movements. To determine the extent of the visual system's contribution to the filter, we performed two types of experiments. First, we performed psychophysical experiments with human subjects to test how well the spatiotemporal filter that we derived from pursuit predicts perceptual judgments of motion direction compared with other filter forms (Fig. 5). Second, we recorded MT unit activity in nonhuman primates to compare the temporal filters for motion integration in cortical neurons mediating pursuit with the behavior itself (Fig. 6).

The motion filter must arise from the joint activity of a population of cortical neurons, each with a limited RF. Although the collective spatial filter is difficult to access physiologically, perceptual estimates are often used as a proxy for internal sensory estimates. To determine whether the filter that we compute from eye movements represents visual processing shared with perception, we quantified how well the pursuit filter predicts subjects' perceptual choices compared with other filter shapes. We performed parallel pursuit and perception experiments in two human subjects $(\mathrm{H} 1, \mathrm{H} 3)$ using a $30^{\circ}$-diameter noisy dots stimulus. In the $2 \mathrm{AFC}$ perceptual discrimination experiment, subjects were asked to indicate whether the average stimulus direction over the $250 \mathrm{~ms}$ motion interval was upward or downward with respect to horizontal (see Materials and Methods). We assigned responses 
+1 for upward and -1 for downward choices. To put the eye movement data on the same footing as the binary perceptual reports, we averaged the eye direction in a $5 \mathrm{~ms}$ time window centered at $250 \mathrm{~ms}$ and assigned the value $\mathrm{a}+1$ if rotated upward and -1 if downward. We only analyzed trials in which the timeaveraged stimulus direction was horizontal $\left(0^{\circ}\right.$ or $\left.180^{\circ}\right)$. See Materials and Methods for a detailed description of the experiments and analysis.

The filter that we compute from eye movements will be the function that best predicts pursuit, but it may not be the function that best predicts perceptual judgments of motion direction. To find the spatial and temporal filter forms that best predict perceptual judgments of motion direction, we created a set of 209 spatial filters and 208 temporal filters. The filter sets were based on Gaussian fits to the actual spatial, $F(R, \theta)$, and temporal, $F(T)$, pursuit filters, but with different means (peak locations or delays) and widths (FWHM, in visual degrees or ms; see Materials and Methods). The spatial filter peak locations spanned $0.5^{\circ}$ to $9.5^{\circ}$ in $0.5^{\circ}$ (degrees of spatial arc) increments and temporal filter delays ranged from 90 to $240 \mathrm{~ms}$ in $10 \mathrm{~ms}$ increments. The widths (FWHM) for the spatial filter set ranged from $2^{\circ}$ to $9.5^{\circ}$ in $0.75^{\circ}$ increments, or 25 to $85 \mathrm{~ms}$ in $5 \mathrm{~ms}$ increments for the temporal filters. We held $F(\theta)$, the weighting as a function of spatial direction, constant for all tested filter forms. The set of synthetic spatial filters are illustrated in the margins of Figure $5 A$ by colored dashed lines. We used each spatial and temporal filter form to generate trial-by-trial predictions of the behavioral response 250 ms after motion onset by convolving the filter with the dot motions. To quantify the quality of the prediction, we computed the linear correlation coefficient between the predicted and actual (binary) eye directions or perceptual reports (Fig. 3). Figure $5 \mathrm{~A}$ shows linear correlation coefficients for two subjects' pursuit (see Materials and Methods), with spatial filter predictions on the left and temporal filters on the right. Each grayscale element in the density plots corresponds to a single filter form. Lighter shades indicate better prediction. Along each column, the filter peaks at a different location (or delay) and, along each row, the filters have a different width. When changing the spatial filter form, we held the temporal filter fixed and vice versa. The patterns of correlations across the different filter shapes were quite similar for both human subjects $(\mathrm{H} 1, \mathrm{H} 3)$. In each case, the best predictions (Fig. $5 A$, bold red boxes) were made with the actual spatial or temporal pursuit filter, as expected. Subject H1's pursuit filter has a correlation coefficient with binary eye direction of $0.77 \pm 0.04$ (SD) ( $n=100$ random samples of $50 \%$ of total dataset), indicating that the filter accounts for $59 \%$ of the eye direction variance. Subject H3's pursuit filter has a correlation coefficient of $0.78 \pm 0.04$ (SD) $(n=100)$ representing $61 \%$ of the variance. The region of near-white pixels indicates the range of filter forms with a correlation coefficient within 2 SDs of the best prediction. Eye direction estimates were somewhat more sensitive to the shape of the spatial than the temporal filter, seen by the smaller white patch on the left versus the larger white patches on the right in Figure $5 \mathrm{~A}$ for both subjects. The lowest correlation values were 0.44 and $0.46(\mathrm{H} 1, \mathrm{H} 3)$, reflecting the background level of correlations in the predictions due to the similarity in the tested filter shapes and the dot direction statistics (see Materials and Methods). Although the motion of each dot is stochastic, the time-averaged motion direction is the same for all dots. Therefore, averaging dot motions under overlapping filters centered at different spatial locations will produce correlated results by the law of averages and the direction update interval creates correlations in dot directions over time. All matrix elements in Figure $5 \mathrm{~A}$ represent statistically significant correlation coefficients with $p \leq 0.01$ with respect to a random permutation of the pursuit responses. See Materials and Methods for a detailed description of all analyses.

We performed a parallel analysis on the perceptual data from the same subjects. To predict perceptual direction estimates from trial to trial, we used each spatial and temporal filter in the set to estimate the perceived time-averaged motion direction over the $250 \mathrm{~ms}$ interval (see Materials and Methods) and computed the linear correlation coefficient with the actual binary perceptual reports. If perception pools signals over a different spatial region of the stimulus or over a different time interval, then the spatial and temporal filter that best predicts perception may differ from the pursuit filter. However, we found that the pursuit filter was the best predictor of the perceptual data (Fig. $5 B$, red boxes). If all motion directions are analyzed, the pursuit filter predicts perception with comparable predictive power as for pursuit. Subject H1's pursuit filter had a correlation coefficient of $0.76 \pm 0.06$ (SD) ( $n=100$ random samples of $50 \%$ of total dataset) with reported stimulus direction, accounting for $58 \%$ of his perceptual report variance. Subject H3's pursuit filter had a correlation coefficient of $0.77 \pm 0.06$ (SD) $(n=100)$ accounting for $60 \%$ of the perceptual variance. When only the $0^{\circ}$ (rightward) trials are included in the analysis, the fluctuations in stimulus global motion direction $\left(0.05 \pm 0.08^{\circ}(\mathrm{SD}), n=210\right.$ trials $)$ are below human discrimination threshold (de Bruyn and Orban, 1988; Mukherjee et al., 2015; $\sim 1.7^{\circ}$ ), so subjects perform at chance levels. Chance level performance means that perceptual choices will be less well correlated with the stimulus, so no motion filter will predict perceptual choices well. However, restricting the data analysis to the $0^{\circ}$ (rightward) trials is advantageous for minimizing the contribution of the time-averaged motion direction that can create spurious patterns of correlation in Figure $5 B$. Using just $0^{\circ}$ trials, the pursuit filter is still the best predictor of perceptual choice (Fig. 5B, red boxes), with correlation coefficients of $0.26 \pm 0.03(\mathrm{SD})$ for subject $\mathrm{H} 1$ and $0.25 \pm 0.02(\mathrm{SD})$ for subject H3 ( $n=100$ random samples of $50 \%$ of data). All matrix elements in Figure $5 B$ represent statistically significant correlation coefficients with $p \leq 0.01$ with respect to a random permutation of the perceptual responses. The fact that the best predictor of perceptual choices at chance-level performance is the pursuit filter is a strong indication that the spatiotemporal filter that we derive from eye movements reflects visual motion processing that is shared between pursuit and perception.

Prediction quality rolls-off with deformations of the pursuit filter in a similar way for the pursuit and perception data. Both subjects' data showed remarkably similar patterns of correlation for pursuit and perception for different spatial filter forms (Fig. 5A, B, left). Prediction quality falls by $>2$ SD units for shifts in the spatial filter peak of $>1.5$ degrees of spatial arc and for spatial filter widths that are $>2.75^{\circ}$ narrower or $4.25^{\circ}$ wider than the pursuit filter for both subjects' pursuit and perception data (Figs. $5 A, B$, left). Compared with the narrow range of spatial filters that gave good behavioral predictions, a somewhat larger range of temporal filter forms predicted both subjects' pursuit and perceptual responses well. The area of lighter pixels is larger in the right compared with the left panels in Figure 5, $A$ and $B$. Pursuit and perception prediction quality were within 2 SD units of the peak over a $40 \mathrm{~ms}$ range of temporal filter peak delays for $\mathrm{H} 1$; that is, $-20 \mathrm{~ms}$ to $+20 \mathrm{~ms}$ around $190 \mathrm{~ms}$ for pursuit (Fig. $5 A$, top right) and $-10 \mathrm{~ms}$ to $+30 \mathrm{~ms}$ for perception (Fig. $5 B$, top right). The same $40 \mathrm{~ms}$ range of temporal filter peak delays $(-10 \mathrm{~ms}$ to $+30 \mathrm{~ms}$ ) predicted H3's perceptual choices well (Fig. $5 B$, bottom right), but a larger range of filter delays predicted H3's pursuit within 2 SD units of the highest correlation coefficient 
( $-40 \mathrm{~ms}$ to $+40 \mathrm{~ms}$; Fig. $5 A$, bottom right). Overall, the changes in behavioral prediction quality with deformations of the spatial and temporal filter shapes were consistent between pursuit and perception data and between subjects.

We summarized the similarity in the predictions of pursuit and perceptual responses by plotting the distributions of correlation coefficients. The histogram plots in Figure $5 C$ represent the distributions of all correlation coefficient values in Figure 5, A and $B$, converted to $z$-scores by subtracting the mean and normalizing by the SD within each panel. The extent to which the distributions for the pursuit versus perceptual data overlap indicates the similarity in filter performance. For both subjects, the span of spatial filter forms with good prediction performance was similar for pursuit and perception (RMS variance of $z$-scored correlation coefficients; H1: $1.3 z$-score units for pursuit, 1.0 for perception, top left; H3: 1.2 pursuit, 1.1 perception, bottom left; Fig. $5 C$ ). The span of temporal filter forms that predict pursuit and perception well is also similar, but less so than for the spatial filter predictions (RMS variance of $z$-scored correlation coefficients; H1: $1.8 z$-score units pursuit, 1.2 perception, top right panel; H3: 1.7 pursuit, 1.2 perception; Fig. $5 C$, bottom right) for both subjects. The similarity in temporal filtering for pursuit and perception is not expected because the dynamics of generating the eye's responses need not match the time scale of generating perceptual reports from visual estimates. Therefore, two pieces of evidence support a visual origin for the spatiotemporal filter. The best predictor of perceptual performance is the pursuit filter and deforming the pursuit filter degrades prediction quality for both pursuit and perception in the same way.

\section{Temporal integration in MT neurons versus pursuit}

To further test the hypothesis that the motion filter that we computed from pursuit eye movements arises primarily from visual rather than motor processing, we compared temporal motion filtering in MT with pursuit. We performed parallel pursuit and neurophysiology experiments to compare temporal filters for motion integration in single MT neurons with pursuit eye movements in monkeys. We used both the noisy dots stimuli and coherent motion versions of those stimuli that shared the same temporal statistics but without the variation across space (see Materials and Methods). For the coherent stimuli, all dots updated to the same randomly drawn direction value every 20 or 40 $\mathrm{ms}$ to produce time-varying motion (see Materials and Methods). We found no significant differences in neural filters based on stimulus form. We projected motion stimuli onto each unit's excitatory RF while the monkeys fixated. We rotated the timeaveraged or base directions of stimulus motion to the flanks of the neuron's direction tuning curve where motion sensitivity is largest. In this way, we made several measurements of the temporal filter for each recorded MT unit. We computed neural temporal filters by relating spike count and motion direction over time using the same analysis technique as described for eye movements. We counted spikes in overlapping $20 \mathrm{~ms}$ time windows and then computed the correlation between the count values and motion direction at $1 \mathrm{~ms}$ intervals up to lags of $200 \mathrm{~ms}$. The temporal filters for three example MT neurons from two monkeys are plotted in Figure $6 B$ (bottom). We also used the translating versions of the same stimuli to compute pursuit temporal filters, shown in Figure 6B, top. The temporal filter forms were quite similar between individual MT units and pursuit behavior, although the MT filters have shorter peak delays than pursuit, as expected. Figure $6 C$, top, shows the distribution of filter durations (FWHM) for the MT units (red trace) and pursuit data (black trace). The population-mean width of the pursuit temporal filters is $29 \pm 4 \mathrm{~ms}$ (SD, $n=96$ datasets, M2-M4), only $5 \mathrm{~ms}$ shorter than the population mean for the MT sample, $34 \pm 12 \mathrm{~ms}$ (SD, $n=138$ measurements from 44 neurons, M3-M4). The distributions of neural and behavioral temporal filter durations are highly overlapped though still statistically distinct with a $p<$ $3.1 \times 10^{-5}$ (two-sample $t$ test). The distributions of peak delays are also similar in the MT and pursuit data. The population mean peak delay of the MT neurons was $73 \pm 7 \mathrm{~ms}(\mathrm{SD}, n=138$ measurements from 44 neurons, M2-3) and for pursuit was $92 \pm$ $6 \mathrm{~ms}$ (SD, $n=96$ datasets, M2-M4; bottom panel; Fig. $6 \mathrm{C}$ ). The overall latency shift between the neural and behavioral filters is expected, but what is notable is that the range of delays is similar for pursuit and MT neurons. If we shift the behavioral distribution to match the mean delays (Fig. 6C, red dashed line, bottom), then the MT and pursuit distributions of peak filter delays overlap ( $p=1$, two-sample $t$ test). Our results suggest that pursuit could be driven by the full range of MT neurons, as could motion perception presumably. Therefore, we cannot explain the different pattern of temporal filter predictions for pursuit and perception in Figure 5 from obvious differences in cortical motion inputs. These results do not rule out contributions from downstream visual or motor processing to the form of the pursuit motion filter (Grinvald et al., 1994; Sincich et al., 2009; Butts et al., 2011), but they suggest that little such refinement is necessary to account for pursuit.

Our experiments were not designed to test response scaling with RF location because we sized the motion stimuli to the RF of each MT unit. Nevertheless, we tested for neural filter differences based on RF eccentricity. We found no statistical dependencies in either neural filter amplitude $\left(R^{2}=0.1, n=115\right.$ datasets from 44 neurons) or duration $\left(R^{2}=2 \times 10^{-4}, n=115\right)$ based on RF location. Because we did not use the same stimulus aperture sizes for the neurophysiological recordings, it is difficult to interpret the lack of eccentricity dependence of MT filters in terms of the spatial motion filters for pursuit and perception.

\section{Dynamics in motion integration for pursuit}

How motion is integrated across space need not be the same over time. Dynamics in MT neural responses, downstream motor processing, or execution of the eye movement itself could all potentially alter the form of the measured motion filter over time (Lisberger and Movshon, 1999; Osborne et al., 2004). We tested the stationarity of motion integration by computing the pursuit filter in different time intervals of the eye movement response (Fig. $7 \mathrm{~A}$, inset). Here, we only analyzed experiments with $30^{\circ}$ diameter noisy dot stimuli. We computed the spatiotemporal filter in overlapping $150 \mathrm{~ms}$ time windows spanning the first 450 ms of pursuit (Fig. 7A) using the spatial segmentation described in Figure 2. We were able to compute motion filters throughout the first $300 \mathrm{~ms}$ of pursuit, but at later times, the filter calculation became unstable, perhaps because of the diminishing differences between the eye and target direction with ongoing pursuit.

We find that the directional anisotropy in the pursuit spatial filter amplitude is highly dynamic, but other features of the spatial filter are less so. The preferential weighting of motion in spatial locations along the direction of motion is transient in monkeys and humans. We plotted the amplitude of subject M1's spatial filter as a polar function of spatial direction segment in Figure $7 B$. Each connected curve represents a different $150 \mathrm{~ms}$ time window, colored as per Figure $7 A$. We found that the direction bias is strongest at pursuit onset, when motion in the spatial regions "downstream" of the fovea along the direction of motion 
A

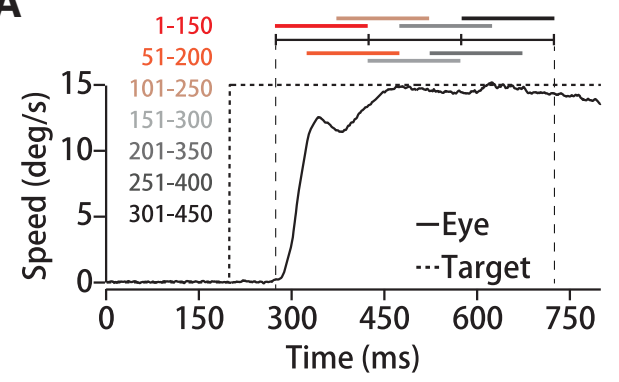

B
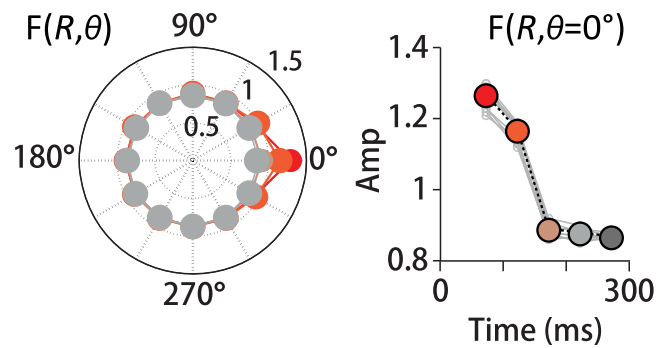
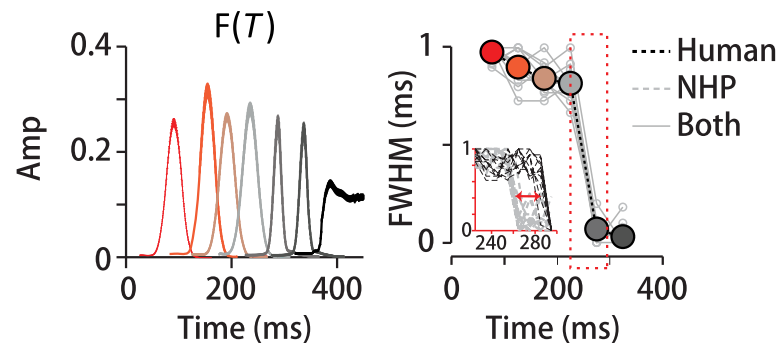

Figure 7. Dynamics of spatial and temporal motion integration. $\boldsymbol{A}$, Target (dotted line) and eye speed (solid line) over time. Diagram indicates the overlapping $150 \mathrm{~ms}$ time windows in which we computed filters. $\boldsymbol{B}$, Left, Polar plot of the directional dependence of the peak filter amplitude in the time windows defined in $\boldsymbol{A}$ for $\mathrm{M} 1$. The directional bias is strongest early in pursuit (red-orange symbols), diminishing to uniform weighting at $175 \mathrm{~ms}$ (red-gray symbols). Right, Time evolution of peak filter amplitude in the $\theta=0^{\circ}$ segment in front of the eye. Colored circles indicate mean values, thin gray lines data from 12 datasets (M1-M2, H1-H3). C, Filter widths for the spatial direction segment in front of the eye, $F\left(R, \theta=0^{\circ}\right)$, do not change over the time period. Left, Example traces (M1). Right, Population mean spatial filter FWHM ( $n=12$ datasets, M1-M2, H1-H3) as a function of time window center (colored circles). Error SDs are smaller than the symbol size. Gray lines represent individual datasets. D, Left, Example of the time evolution of the temporal filter, $F(T)$ (M1). Right, Filter duration (FWHM; $n=12$ datasets, M1-M2, H1-H3) is stable over the first $225 \mathrm{~ms}$ of pursuit, but narrows in later time windows. Error SDs are smaller than symbol size. Inset expands the area surrounded by the red dotted line. Upping the time resolution to $10 \mathrm{~ms}$ from 50 ms intervals reveals a species difference in the time at which the temporal filters narrow (red arrow). Temporal filter duration (FWHM) drops at $260 \pm 6 \mathrm{~ms}$ (SD) ( $n=8$ datasets, M1-M2) in monkeys (gray dotted lines) and $295 \pm 8 \mathrm{~ms}$ (SD) ( $n=4$ datasets, H1-H3) in humans (black dotted lines).

are weighted most strongly (also see Fig. 2E). The anisotropy decays to rotationally uniform weighting over time. We quantified the deviation from circularity across the population by computing the circular variance in the peak filter amplitude in each time window. Higher values of the circular variance indicate more directional anisotropy. The circular variance decreased from $0.26 \pm 0.07(\mathrm{SD})(n=12$ datasets, 5 subjects, M1-M2, $\mathrm{H} 1-\mathrm{H} 3)$ in the earliest time window centered at $75 \mathrm{~ms}$ to $0.18 \pm$ $0.07(\mathrm{SD})$ at $275 \mathrm{~ms}\left(p<6 \times 10^{-9}\right.$, two-sample $t$ test, $\left.n=12\right)$. For both the monkeys and humans, spatial filters became rotationally invariant in time windows starting later than $100 \mathrm{~ms}$ after pursuit onset (centered at $\geq 175 \mathrm{~ms}$ ).

Although the spatial filter's amplitude along the direction of motion changes during pursuit, other features are stable over time. For example, the eccentricity at which the spatial filter peaks has the same value in all spatial direction segments throughout the first $300 \mathrm{~ms}$ of pursuit. We plot the spatial filters for just the $0^{\circ}$-segment over time in Figure $7 C$ (left) for all 12 datasets (M1$\mathrm{M} 2, \mathrm{H} 1-\mathrm{H} 3)$, showing that the spatial filters nearly superimpose when we normalize to the peak amplitude. The differences in spatial filter peak eccentricity were not statistically significant across all monkey and human datasets (monkeys $p=0.92, n=$ 576, 12 directions, 8 datasets, and 6 time windows; one-way ANOVA; humans $p=0.98, n=288,12$ directions, 4 datasets, 6 time windows; one-way ANOVA). The width of the $0^{\circ}$-segment's spatial filter (FWHM) is also constant over the first $300 \mathrm{~ms}$ of pursuit ( $p=0.28, n=12$ datasets from 5 subjects, paired $t$ test), but it remains significantly narrower than the spatial filter for all except the adjacent direction segments even when filter amplitudes are normalized. We plot the spatial filter width (FWHM) for the $0^{\circ}$-segment (gray lines) and the $180^{\circ}$-segment (black dot- ted lines) as a function of time window center for all datasets and subjects in Figure $7 C$, right. The closed circles represent the sample mean (FWHM of the $0^{\circ}$-segment spatial filters) and are colored by time window identity according to Figure $7 A$. The closed circles occlude the individual dataset values somewhat (gray lines) because they are tightly clustered. The difference between the FWHM of the $0^{\circ}$-segment and $180^{\circ}$ segment filters was $-0.5 \pm 0.2$ degrees of spatial $\operatorname{arc}(\mathrm{SD}, n=$ 12 datasets from 5 subjects, $p=4.3 \times 10^{-22}$, paired $t$ test). Together, these data suggest that global motion estimates are transiently more sensitive to motion in downstream locations along the motion axis, but that the pooling region is smaller there compared with elsewhere in the visual field for a more sustained period.

The temporal filter is stable across the first $300 \mathrm{~ms}$ of pursuit, but the peak delay decreases and filter duration narrows later in the eye movement. Example data from M1 are plotted in Figure $7 D$, left. In the example data and across monkey datasets, we found that the temporal filter peak delay decreases gradually from $94 \pm 3 \mathrm{~ms}$ (SD) to $87 \pm 2 \mathrm{~ms}$ (SD) ( $n=8$ datasets, M1-M2) between the first time window centered at $75 \mathrm{~ms}$ (Fig. $7 D$, red trace, left) and the last time window centered at $325 \mathrm{~ms}$ (Fig. 7D, dark gray trace, left). For humans, the peak delay changes from $98 \pm 5 \mathrm{~ms}(\mathrm{SD})$ to $88 \pm 6 \mathrm{~ms}$ across the same time interval $(n=$ 4 datasets, H1-H3). The temporal filter duration (FWHM), a measure of the motion integration time, also decreases after $\sim 275$ ms of pursuit, but the transition is more abrupt than the change in peak delay (Fig. 7D). Monkey temporal filter durations are $28 \pm 0.8 \mathrm{~ms}(\mathrm{SD})$ for early time windows $(n=8$ datasets, M1-M2), narrowing to $18 \pm 1 \mathrm{~ms}(\mathrm{SD})$ ( $n=8$ datasets, M1-M2; gray lines, Fig. $7 D$, right). Human temporal filter durations drop 
A

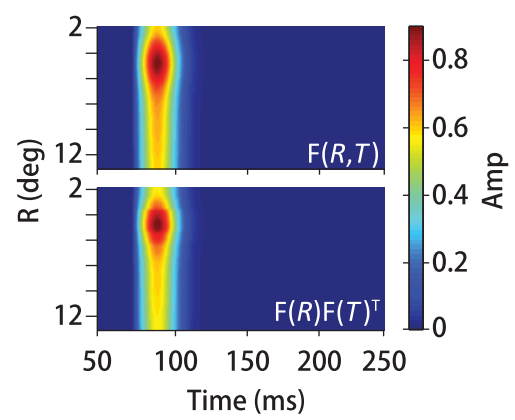

B

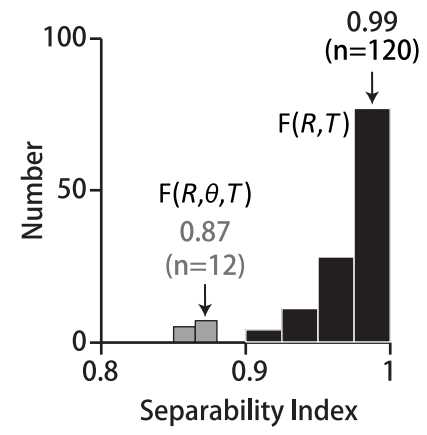

Figure 8. Separability of spatial and temporal motion integration. $A, 2 D$, rotationally averaged filter, $F(R, T)$, is well approximated by the outer product of separate 1D spatial and temporal filters, $F(R) F(T)^{\top}$, seen in the similar color patterns in the top and bottom panels. Data from subject M1 are shown. $\boldsymbol{B}$, Population distribution of separability index values for the rotationally averaged filter $F(R, T)$ (black) and the full filter $F(R, \theta, T)$ (gray). Although $F(R, T)$ is separable $(S I=0.98 \pm 0.04, n=120)$, the full motion filter, $F(R, \theta, T)$, is not $(S I=0.87 \pm 0.04, n=12$ ). Changes in $F(\theta)$ over time link spatial and temporal motion processing.

from $39 \pm 2 \mathrm{~ms}$ to $29 \pm 1 \mathrm{~ms}(\mathrm{SD})(n=4$ datasets, $\mathrm{H} 1-\mathrm{H} 3$; gray lines, Fig. $7 D$, right). To better resolve the times at which each subject's temporal filter narrows, we computed the temporal filters in $150 \mathrm{~ms}$ time windows centered at $10 \mathrm{~ms}$ intervals throughout the pursuit response. The drop in temporal filter duration occurs at $260 \pm 6 \mathrm{~ms}(\mathrm{SD})(n=8$ datasets, M1-M2) in monkeys (gray dotted lines, right panel inset, Fig. 7D) and $295 \pm 8 \mathrm{~ms}$ (SD) ( $n=4$ datasets, $\mathrm{H} 1-\mathrm{H} 3$ ) in humans (Fig. $7 \mathrm{D}$, black dotted lines, right inset). The transition in temporal filter duration and the change in peak delay over time may be due to a switch from open-loop to closed-loop control that is thought to happen earlier in monkeys than in humans (Lisberger and Westbrook, 1985; Goldreich et al., 1992; Churchland and Lisberger, 2001). However, the abrupt shift in filter duration suggests a sharp transition between control states rather than a gradual takeover of nonvisual signals in the oculomotor control loop (Churchland and Lisberger, 2001). Another possibility is that the dynamics might arise from visual processing, caused perhaps by sharpening in MT temporal filters over time. To test this idea, we performed the same sliding time-window analysis on the MT neural data and found that neural temporal filters do not display changes in duration over time. We defined the time windows for the physiological analysis with respect to the average response latency of each unit. We computed the difference in the FWHM of the temporal filter computed in the first time window (1-150 ms) and the last time window (300-450 ms) across our sample. We found that neural filter durations did not change significantly, measuring $36 \pm 15 \mathrm{~ms}(\mathrm{SD})(n=$ 74 measurements from $n=44$ neurons) in the first time window and $34 \pm 14 \mathrm{~ms}(\mathrm{SD})$ in the last time window $(p=0.41$, rank sum test, two-tailed). The stability of MT neural filters over time suggests that the narrowing that we observed in pursuit temporal filters after the first $200 \mathrm{~ms}$ of pursuit has a nonvisual origin.

The extent to which the motion filter changes over time determines how well the 3D spatiotemporal filter can be described as space-time separable. We quantified the filter's separability by computing a separability index (SI) using singular value decomposition (DeAngelis et al., 1992; Peña and Konishi, 2001; Grunewald and Skoumbourdis, 2004; Malone and Ringach, 2008; see Materials and Methods). We analyzed the space-time separability of both the 2D filter $F(R, T)$ and the full 3D filter, $F(R, \theta, T)$. We found that, although the $2 \mathrm{D}$ filter, $F(R, T)$, is separable $(\mathrm{SI}=0.98 \pm 0.04 \mathrm{SD}, n=120$ datasets, M1-M2, H1$\mathrm{H} 3$ ), the full spatiotemporal filter, $F(R, \theta, T)$ is not $(\mathrm{SI}=0.87 \pm$
$0.04 \mathrm{SD}, n=12$ datasets, M1-M2, H1$\mathrm{H} 3)$. We illustrate the separability of the $2 \mathrm{D}$ filter $F(R, T)$ in Figure 8 . The color density representation of filter amplitude is very similar between the actual filter $F(R, T)$ and the outer product of two single parameter filters, $F(R) F(T)^{T}$, where the " $T$ " superscript indicates a transpose operation. The reduced separability of the full motion filter is consistent with the dynamics that we observed in the directional anisotropy of the spatial filter.

\section{Dependence based on stimulus size}

The spatial or temporal span over which motion signals are pooled might adapt to the visual scale of the moving object. We tested for stimulus-size adaptability in spatial motion filtering by changing the aperture diameter of our noisy dot direction targets from 5 to $30^{\circ}$ holding dot density fixed (Fig. $9 A$, colored circles). We computed $F(R)$ in the same manner described previously by dividing the visual field into 59 foveacentered annuli and computing the per-dot contribution at each eccentricity. Each colored curve in Figure $9 B$ represents the spatial filter for the corresponding colored stimulus size. The spatial filter peaks at more eccentric values with increasing aperture size. For the smallest aperture ( $5^{\circ}$ diameter, red trace), the spatial filter peaks close to the fovea $\left(\sim 1^{\circ}\right)$, but the larger the stimulus, the more eccentric the peak (up to $4.75^{\circ}$ for a $30^{\circ}$-diameter aperture). The location of the filter peak scales nearly linearly with target radius such that the peak amplitude falls at $\sim 1 / 3$ of the distance between the fovea and the aperture boundary. The linear fit in Figure $9 C$ has a slope of 0.31 with an $R^{2}$ value of $0.94(n=10$ datasets from $\mathrm{M} 1-\mathrm{M} 2$ ). Note that $F(R)$ is always positive, implying a lack of inhibition from peripheral motion signals often termed surround suppression. This is consistent with studies of spatial motion integration of dot pattern stimuli in perception (Linares et al., 2012) and pursuit (Heinen and Watamaniuk, 1998). The temporal filters were nearly identical for all aperture sizes for 2 monkeys (FWHM $=27 \pm 5 \mathrm{~ms}$ (SD), $n=114$ datasets, M1-M2; $p>0.9$ two-sided Wilcoxon rank-sum test).

We did not have the statistical power to estimate the 3D spatiotemporal filter for the smaller noisy dot stimuli (see Materials and Methods), so we could not evaluate directly whether the directional anisotropy in motion integration depends on stimulus size. As displayed in Figure $3 B$, bottom, the pseudo-3D filter, $F(R, T) \otimes F(\theta)$, accounts for only $2 \%$ more variance on average than the $2 \mathrm{D}$ filter, $F(R, T)$ for stimulus diameters $<30^{\circ}$. To determine whether another function of spatial direction would yield better predictive power for smaller motion stimuli, we tested a range of direction weights. We contracted the number of spatial direction segments to four and then allowed the filter amplitude in each segment to change by $0 \%, \pm 25 \%, \pm 50 \%$, and $\pm 75 \%$ ( $7^{4}$ combinations) and computed the correlation coefficient between the predicted and actual global motion direction over time for each model $F(\theta)$. We found that the best predictor of pursuit was the function $F(\theta)$ measured from the $30^{\circ}$ stimuli. Although this analysis is not conclusive, it does suggest that the directional anisotropy in motion weighting is not dependent on stimulus size. 
Optic flow content predicts pursuit gain for novel stimuli

There are many reasons why the motion filter might vary for different visual stimuli. Differences in spatial and temporal frequency content, contrast, and the strength of the optic flow might drive different patterns and degrees of cortical activation, leading to different stimulusbehavior relationships. Ideally, we would compute a filter for a variety of stimulus forms, but many commonly used laboratory stimuli such as drifting gratings or coherent dot patterns do not have the spatiotemporal variation necessary for the analysis. To test the generality and predictive power of the filter we compute from the spatiotemporal noise stimulus, we investigated how well the filter predicts the pursuit response to different stimuli.

Pursuit gain varies as a function of stimulus size, contrast, coherence, etc.- -all factors that affect motion energy (Lisberger et al., 1987; Heinen and Watamaniuk, 1998; Schütz et al., 2010; Yang et al., 2012; Mukherjee et al., 2015). In previous work, we quantified motion energy by measuring the movement of pixels across adjacent frames weighted by their luminance (see Materials and Methods, Sun et al., 2010; Mukherjee et al., 2015). Here, we introduce the additional step of convolving the filter, $F(R, \theta, T)$, with its optic flow field to create an optic flow score to represent the effective motion energy of the stimulus. As long as the filter, or rather set of filters for different noisy dots stimulus sizes, remains descriptive of internal motion estimates, the flow score should predict the effective drive for pursuit initiation for each target form. The logic underlying this analysis is that the time course of smooth eye acceleration can be predicted from motiondriven cortical activity (Kawano et al., 1994; Lisberger and Movshon, 1999) and therefore, if the filter represents the interpretation of the cortical population activity, then it may predict the gain of the pursuit response. We tested this hypothesis by comparing the predicted and measured pursuit gains for a variety of motion stimuli with a fivefold range in flow scores. To generate a flow score for each stimulus, we used a single set of 2D filters, $F(R, T)$, computed from noisy dots experiments with a dot density of $1 \mathrm{dot} / \mathrm{deg}^{2}$ and speed of $15 \%$ displayed in Figure 9B. Because we could only compute the full, 3D motion filter, $F(R, \theta, T)$, with the $30^{\circ}$ noisy dots stimulus, we had to assume that the pattern of directional weighting in time was consistent across aperture sizes to generate flow scores. With two exceptions ( $2^{\circ}$ dot pattern, spot target), we generated new stimuli with the same aperture diameters $\left(5^{\circ}, 7^{\circ}, 10^{\circ}, 14^{\circ}\right)$ for which we had measured spatiotemporal filters to minimize the need for interpolation. For the two small stimuli, we shifted the $5^{\circ}$ stimulus filter to the peak location predicted by the linear relationship plotted in Figure 9 C.

Figure $10 \mathrm{~A}$ shows an example of how stimulus motion energy affects the initiation of pursuit. Peak eye acceleration in response to a motion step is generally higher for a high-score stimulus (black dotted trace) than a low-score stimulus (black solid trace),
B
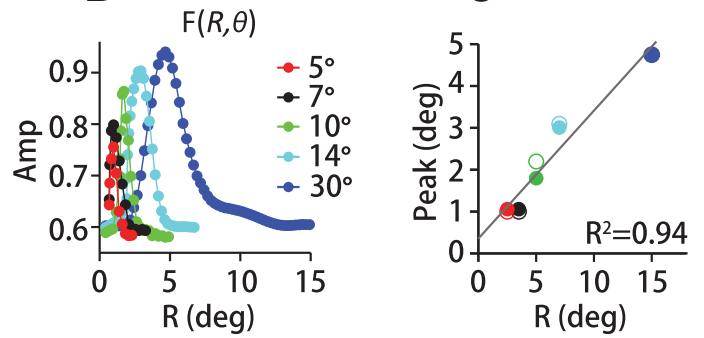

Figure 9. Spatial motion integration depends on stimulus size. $\boldsymbol{A}$, We tested stimulus diameters from 5 to $30^{\circ}$, indicated by the eccentric the filter's peak. $C$, Foveal eccentricity of the spatial filter's peak scales linearly with stimulus radius, $(f(x)=0.30 x+$

Figure 10. Motion filter predicts the effective drive for pursuit across several stimulus forms. Convolving the motion filter with (henerates a scalar optic flow score related to the visual drive for pursuit. $\boldsymbol{A}$, Trial-averaged eye speed (top) and acceleracceleration) is proportional to the flow score. Thirty-three closed black circles (symbol group 1) represent pursuit experiments for and accounts for $96 \%$ of the variance in pursuit gain $\left(R^{2}=0.96, n=33\right.$ group 1 stimuli, M1-M2, H1-H3). Pursuit gains for additional stimuli are plotted in groups (group $2=$ coherent dot motion, $3=$ Gabors, $4=$ checkerboards; see text). A line of explains $83 \%$ of the variance for all but 2 tested stimuli (red dashed line, $n=48$ ). Pursuit gains for two stimulus forms were not well predicted by flow score (and thus by the noisy dots motion filter): a small spot target (open green circles) and a Gabor that translated against a dark background (see text, blue open circles). Using unfiltered dot motion to generate a flow score for the spot target placed it within the fitted stimulus forms (green closed circles).

even though the average eye speeds after $200 \mathrm{~ms}$ of pursuit are nearly identical. The trial-averaged eye movements in Figure $10 \mathrm{~A}$ are both from experiments with $15 \%$ s noisy dots targets with the same dot density but different aperture sizes. The sinusoidal ringing in the eye speed trace for the high-score stimulus (Fig. 10A, black dotted trace) is the predicted result of an increase in openloop gain (Churchland and Lisberger, 2001). Drawing on experiments with noisy dot stimuli of different sizes $\left(5-30^{\circ}\right)$, dot densities $\left(1,2\right.$, and 4 dots $\left./ \mathrm{deg}^{2}\right)$, and speeds $\left(10-20^{\circ} / \mathrm{s}\right)$, we performed a linear regression between the measured trial-averaged peak eye acceleration and optic flow score (Fig. 10B, solid black circles and solid red line). Each symbol in Figure $10 B$ represents the average pursuit gain over multiple datasets with the same stimulus and subject. With the noisy dot experiments alone, a linear regression explains $96 \%$ of the gain variance (solid red line, $R^{2}=0.96, n=33$, M1-M2, H1-H3; Fig. 10B). We performed additional pursuit experiments with alternate stimulus forms to determine whether the motion filter derived for the noisy dots targets also predicts pursuit gain for different motion stimuli (Fig. 10B, colored circles). See Materials and Methods for a complete description of each stimulus. We found that pursuit gain was well predicted for all but two of the stimuli. The eight solid 
circles in shades of orange to brown (symbol group \#2; Fig. 10B) represent coherent-motion random dot patterns with different aperture diameters $\left(5^{\circ}-14^{\circ}\right)$. Dot density was $1 \mathrm{dot} / \mathrm{deg}^{2}$ and dot speed was $15^{\circ}$ s for all experiments within this group (M1, M2). The five solid circles in shades of blue represent variations on Gabors (M1, M2). The darkest blue shade is a $14^{\circ}$-diameter Gabor $\left(100 \%\right.$ contrast, $\left.\mathrm{SD}=2.75^{\circ} ; 1 \mathrm{cycle} / \mathrm{deg}\right)$ that translated at $15 \%$ against a gray background set to the mean luminance of the target (see Materials and Methods). The medium blue symbols represent a $5^{\circ}$-diameter Gabor $\left(\mathrm{SD}=1.2^{\circ}\right)$ that was otherwise identical. Cyan circles represent an array of $13,2^{\circ}$-diameter $\mathrm{Ga}$ bors $\left(S D=0.75^{\circ}\right)$ spaced $1-2^{\circ}$ apart to create a target diameter of about $14^{\circ}$. The magenta circles represent experiments with a $14^{\circ}$ checkerboard pattern of $1.8^{\circ}$ alternating black and white squares (M1, M2). A linear regression that includes both the noisy dots data (black circles) and the new stimuli is very similar to the relationship derived for the noisy dots data alone (see legend in Fig. $10 B$ ) and explains $83 \%$ of the gain variation (dashed red line, $R^{2}=0.83, n=48$ data points, M1-M2, H1-H3).

The motion filter did not predict pursuit gain for two stimulus forms (Fig. 10B, open circles, symbol group \#5). One of those targets was a $0.25^{\circ}$ white circular spot target translating at $16^{\circ} / \mathrm{s}$ (M1, M3), represented by the green circles in Figure 10B. The spot target is so small with respect to the dot pattern stimuli from which we measured the filter we simply weighted each pixel equally (open green circles; Fig. 10B). We extrapolated the weight value from the linear relationship between peak filter amplitude and aperture radius presented in Figure 9C. If we do not apply any scaling to the spot target's flow score, that is, score $=$ pixel speed $\times$ number of illuminated pixels, then the pursuit gains of M1 and M2 are well predicted by the linear fits in Figure $10 B$ (solid green circles). It is therefore unlikely that the motion filter that we derived from dot stimuli generalizes to spot targets. Finally, blue open circles represent a translating Gabor, identical to the first Gabor described above (darkest blue, symbol group \#3) except that the background luminance was set to zero instead of the average grayscale level. Background luminance affects the total pixel flow in our algorithm (see Materials and Methods) and thus the flow scores for the two Gabors are somewhat different. However, the pursuit gains are very different. Eye acceleration is substantially higher for a Gabor translating against a dark background (open dark blue circles) compared with a gray background (open dark blue circles; Fig. 10B, M1, M2). Overall we found that, with the exception of the spot and nonzero background Gabor stimuli, a linear model of spatiotemporal direction estimation explains a large fraction of the variance in pursuit gain for multiple visual stimuli. The predictive power is impressive given that the filter relates eye to stimulus direction over time, yet here we have used the filter to predict the relationship between motion energy and pursuit gain.

In smooth pursuit, peak eye acceleration is linearly related to target speed for the speed range used in these experiments (Lisberger and Westbrook, 1985). Could low eye accelerations arise because the brain underestimates the speed of low score stimuli? We tested this idea by measuring the time-averaged eye speed in a $50 \mathrm{~ms}$ window from 150 to $200 \mathrm{~ms}$ after pursuit onset and computing the difference between eye speed and stimulus speed. If speed estimation errors are correlated with flow score, then we would expect to see larger, more negative errors for low score stimuli and decreasing error magnitude as flow scores increase. However, we did not detect a correlation between speed error and flow score in either of our monkey's data $\left(R^{2} \leq 0.02, n=20 \mathrm{dot}\right.$ stimulus experiments, 10 with scores $<3 \times 10^{5}$ and 10 with scores $\left.>6 \times 10^{5} ; \mathrm{M} 1, \mathrm{M} 2\right)$. Neither subject's linear regression explained enough the variance across stimuli to motivate a Bayesian model of speed estimation.

\section{Discussion}

We have focused on short-timescale integration of random dot pattern motion studied through the lens of pursuit eye movements. The spatiotemporal filter captures how the movements of point objects are integrated over space and time to estimate direction (Williams and Sekuler, 1984; Watamaniuk et al., 1989, 2011; Smith et al., 1994). Although we use eye movements to derive the filter, visual and motor processing specific to pursuit do not appear to influence its form. Rather, we found that the same filter predicts both pursuit and perceptual judgments of motion direction. Consistent with past studies of global motion perception (Watamaniuk and Sekuler, 1992; Smith et al., 1994) and pursuit (Heinen and Watamaniuk, 1998), we found that all motion within the visual field adds. The literature suggests, however, that spatial motion integration is stimulus dependent because large-sinewave gratings decrease the internal signal-tonoise ratio (Tadin and Lapin, 2005; Tadin et al., 2003), whereas large dot fields increase it (Linares et al., 2012). The spatial filter's eccentric peak $\left(\geq 1^{\circ}\right)$ and non-negative profile are consistent with several psychophysical studies of dot motion that found low perceptual sensitivity for near-foveal motion, then rapid improvement and leveling off with increasing stimulus size (Gorea, 1985; Anderson and Burr, 1987; Watamaniuk and Sekuler, 1992; Fredericksen et al., 1994; Morrone et al., 1995; Watson and Turano, 1995). Our spatial calculations are similar to Neri and Levi (2009), who divided their stimulus aperture into annuli and computed stimulus-report correlations in each division. They found that motion was weighted fairly uniformly across an eccentrically located $2^{\circ}$ aperture; however, their study's surround distractor complicates comparison with our results because surround motion can affect the area over which motion is perceptually averaged (Sasaki and Uka, 2011).

A striking feature of our spatial filter is its transient directional anisotropy. There is no clear consensus in the psychophysical literature for spatial bias in motion summation based on motion direction (Ball and Sekuler, 1980; Raymond, 1994). There is a physiological analog for our behavioral results, however, from studies of attentional modulation in MT. Spatially directed attention enhances responses in MT neurons with RFs that overlap the attended area (Cook and Maunsell, 2004). If our pursuit and perceptual tasks enhance spatial attention and MT neuron firing rates at locations along the expected direction of motion, then the directional anisotropy that we observed in the motion filter is expected. A later study suggests that spatial attention creates an attractive shift in MT RF centers and narrows RF extent, providing a candidate physiological mechanism for our observation that motion weights are enhanced and the spatial filter narrows along the expected direction of motion. (Womelsdorf et al., 2008)

Motion integration times in pursuit are also consistent with perceptual studies. For large area stimuli, motion is perceivable with $30 \mathrm{~ms}$ presentations (van Doorn and Koenderink, 1984). Tadin et al. (2006) measured a temporal motion filter based on the correlation between stimulus motion and perceptual reports, finding a duration of $\sim 30 \mathrm{~ms}$ consistent with our pursuit-based measurement. These and other studies suggest that pursuit and perception integrate motion over time quite similarly (Kowler and McKee, 1987; Watamaniuk et al., 1989; Osborne et al., 2007, 2009; Mukherjee et al., 2015). The filter's peak delay, however, is more likely a result of pursuit-specific motor processing. Al- 
though the spatial filters that we derived for humans and monkeys are nearly identical, their temporal filters are time shifted by an amount approximately corresponding to the species difference in pursuit latencies. Furthermore, manipulating the peak delay did not affect predictions of perceptual reports, whereas manipulating the duration of the temporal filter did. Given the similarity to filter durations in MT neurons, we suggest that the temporal filter duration that we measured represents visual processing.

Motion integration is highly context dependent. Spatial and temporal summation of motion signals changes with the nature of the stimulus (contrast, spectral content, spatial scale, etc.) and the task (e.g., object segmentation vs heading perception; Braddick, 1993; Tadin et al., 2008; Burr et al., 2009). Stimulus-driven differences in motion integration should therefore give rise to different motion filters. Here, we have analyzed differences in motion integration as a function of stimulus size and found that the form of the spatial filter changes substantially with aperture diameter. The peak eccentricity for motion weighting rescales to $\sim 1 / 3$ of the stimulus radius for each aperture size that we measured. Ideally, we could compute the filter for a range of stimulus forms and tasks to quantify stimulus driven changes in motion summation changes, but many commonly used laboratory stimuli, such as drifting Gabors, lack the spatial and temporal variation to compute a filter. We therefore used the less direct approach of testing for generality in the filter by predicting the gain of the pursuit response for stimuli of different size, coherence levels, and spectral bandwidth. Although far from an exhaustive study, the success of the filter to predict pursuit gain for all but a few targets indicates a measure of generalizability across stimulus forms. However, the filters that we report did not predict the high sensitivity of pursuit and perception of small, moving spot targets and one of the translating Gabors. Nor do they explain why dense stimuli like Gabor patches and speckle patterns of sizes larger than $\sim 1.5^{\circ}$ suppress motion sensitivity ( $\mathrm{Ta}-$ din et al., 2003; Tadin and Lapin, 2005), whereas dot stimuli show the opposite effect (Linares et al., 2012; present study). These failures confirm the expectation generated by diverse and often conflicting results in the literature that the inherent adaptability of motion integration will restrict the degree of generalization of any single model of motion processing (Braddick, 1993). That said, the spatiotemporal filter that we measured from pursuit generates testable predictions of perceptual judgments and motor responses to some alternate stimulus forms. How behavior deviates from filter predictions may provide a clue to the nature of the underlying nonlinearities in motion integration.

Global motion estimation has been described by an averaging operation (Williams and Sekuler, 1984; Watamaniuk et al., 1989, 2011; Watamaniuk and Sekuler, 1992; Smith et al., 1994; Verghese et al., 1999), winner-take-all (Churchland and Lisberger, 2001; Sheliga et al., 2006), and by probabilistic estimation (Stocker and Simoncelli, 2006; Yang et al., 2012). The fact that a linear model can predict pursuit and perception quite well is consistent with vector averaging; however, a strict vector-average would pool all available motion signals. We found that, whereas all motion signals are used, contributions from a narrow eccentricity band are emphasized. It seems counterintuitive that the brain would devalue motion signal from much of the visual field unless it is taken as noise. Past studies have reported spatial interference effects from peripheral motion (Bex et al., 2003; Bex and Dakin, 2005) that drive attention-based contraction of the perceptual pooling area while increasing sensitivity (Sasaki and Uka, 2011). If a similar mechanism is at play here, then our results suggest that the divisive normalization that underlies the vector average can operate on both local (Chang and Julesz, 1983; Morgan, 1992) and global (Williams and Sekuler, 1984; van Doorn and Koenderink, 1984; Watamaniuk et al., 1989; Watamaniuk and Sekuler, 1992; Smith et al., 1994; Verghese et al., 1999; Sheliga et al., 2008) scales. Given the behavioral flexibility in motion integration (Braddick, 1993; Tadin et al., 2008), it seems plausible that normalization operations are either themselves flexible or that their overall contribution to behavior depends on context. Departures from linearity in global motion processing may provide clues to the nature of the normalization.

\section{Evidence for Bayesian estimation}

We used the spatiotemporal filter to investigate the relationship between stimulus optic flow content and pursuit gain. How the eye accelerates initially and the eye speed at the end of the openloop interval is related to internal estimates of target speed (Lisberger and Westbrook, 1985; Lisberger and Movshon, 1999; Osborne et al., 2005). For example, if the brain underestimates the target's speed, the eye's acceleration and steady-state speed will be too slow until visual feedback becomes available to correct pursuit (Lisberger et al., 1981; Lisberger and Westbrook, 1985; Osborne et al., 2005). We found that scoring motion stimuli based on filter-weighted summed optic flow allowed us to predict pursuit gain over a fivefold range of optic flow scores, an indication of the filter's generality. With two exceptions, eye acceleration increases in proportion to flow score. This result is consistent with past studies linking behavioral gain to motion energy (Lisberger and Westbrook, 1985; Heinen and Watamaniuk, 1998; Sheliga et al., 2008a, 2009; Schütz et al., 2010; Mukherjee et al., 2015). Peak eye acceleration increases linearly with target speed (Lisberger and Westbrook, 1985), so lower pursuit gains could also indicate that the brain underestimates the speed of weaker motion energy stimuli. A Bayesian model of speed estimation predicts this bias by postulating that the brain expects a stationary world: a zero-speed prior (Stocker and Simoncelli, 2006; Yang et al., 2012). Priors have a stronger influence on speed estimates at low signal-to-noise, shifting speed estimates of weaker stimuli toward zero. Therefore, Bayesian models predict larger, negative speed errors for low flow score stimuli, which we did not observe. Further study with carefully chosen stimuli that tease apart contextual dependencies on motion drive will be necessary to test Bayesian theories more explicitly.

\section{References}

Anderson SJ, Burr DC (1987) Receptive field size of human motion detection units. Vision Res 27:621-635. CrossRef Medline

Ball K, Sekuler R (1980) Human vision favors centrifugal motion. Perception 9:317-325. CrossRef Medline

Bex PJ, Dakin SC (2005) Spatial interference among moving targets. Vision Res 45:1385-1398. CrossRef Medline

Bex PJ, Dakin SC, Simmers AJ (2003) The shape and size of crowding for moving targets. Vision Res 43:2895-2904. CrossRef Medline

Born RT, Tootell RB (1992) Segregation of global and local motion processing in primate middle temporal visual area. Nature 357:497-499. CrossRef Medline

Born RT, Groh JM, Zhao R, Lukasewycz SJ (2000) Segregation of object and background motion in visual area MT: effects of microstimulation on eye movements. Neuron 26:725-734. CrossRef Medline

Braddick O (1993) Segmentation versus integration in visual motion processing. Trends Neurosci 16:263-268. CrossRef Medline

Britten KH, Newsome WT, Shadlen MN, Celebrini S, Movshon JA (1996) A relationship between behavioral choice and the visual responses of neurons in macaque MT. Vis Neurosci 13:87-100. CrossRef Medline

Burr DC, Baldassi S, Morrone MC, Verghese P (2009) Pooling and segmenting motion signals. Vision Res 49:1065-1072. CrossRef Medline 
Butts DA, Weng C, Jin J, Alonso JM, Paninski L (2011) Temporal precision in the visual pathway through the interplay of excitation and stimulusdriven suppression. J Neurosci 31:11313-11327. CrossRef Medline

Chang JJ, Julesz B (1983) Displacement limits, directional anisotropy and direction versus form discrimination in random-dot cinematograms. Vision Res 23:639-646. CrossRef Medline

Churchland MM, Lisberger SG (2001) Shifts in the population response in the middle temporal visual area parallel perceptual and motor illusions produced by apparent motion. J Neurosci 21:9387-9402. Medline

Cook EP, Maunsell JH (2004) Attentional modulation of motion integration of individual neurons in the middle temporal visual area. J Neurosci 24:7964-7977. CrossRef Medline

DeAngelis GC, Robson JG, Ohzawa I, Freeman RD (1992) Organization of suppression in receptive fields of neurons in cat visual cortex. J Neurophysiol 68:144-163. Medline

De Bruyn B, Orban GA (1988) Human velocity and direction discrimination measured with random dot patterns. Vision Res 28:1323-1335. CrossRef Medline

Fredericksen RE, Verstraten FA, van de Grind WA (1994) Spatial summation and its interaction with the temporal integration mechanism in human motion perception. Vision Res 34:3171-3188. CrossRef Medline

Gibson JJ (1950) The perception of the visual world. Boston: Houghton Mifflin.

Goldreich D, Krauzlis RJ, Lisberger SG (1992) Effect of changing feedback delay on spontaneous oscillations in smooth pursuit eye movements of monkeys. J Neurophysiol 67:625-638. Medline

Gorea A (1985) Spatial integration characteristics in motion detection and direction identification. Spat Vis 1:85-102. CrossRef Medline

Grinvald A, Lieke EE, Frostig RD, Hildesheim R (1994) Cortical pointspread function and long-range lateral interactions revealed by real-time optical imaging of macaque monkey primary visual cortex. J Neurosci 14:2545-2568. Medline

Groh JM, Born RT, Newsome WT (1997) How is a sensory map read out? Effects of microstimulation in visual area MT on saccades and smooth pursuit eye movements. J Neurosci 17:4312-4330. Medline

Grunewald A, Skoumbourdis EK (2004) The integration of multiple stimulus features by V1 neurons. J Neurosci 24:9185-9194. CrossRef Medline

Heinen SJ, Watamaniuk SN (1998) Spatial integration in human smooth pursuit. Vision Res 38:3785-3794. CrossRef Medline

Heinen SJ, Badler JB, Ting W (2005) Timing and velocity randomization similarly affect anticipatory pursuit. J Vis 5:493-503. Medline

Hohl SS, Lisberger SG (2011) Representation of perceptually invisible image motion in extrastriate visual area MT of macaque monkeys. J Neurosci 31:16561-16569. CrossRef Medline

Hohl SS, Chaisanguanthum KS, Lisberger SG (2013) Sensory population decoding for visually guided movements. Neuron 79:167-179. CrossRef Medline

Kawano K, Shidara M, Watanabe Y, Yamane S (1994) Neural activity in cortical area MST of alert monkey during ocular following responses. J Neurophysiol 71:2305-2324. Medline

Komatsu H, Wurtz RH (1989) Modulation of pursuit eye movements by stimulation of cortical areas MT and MST. J Neurophysiol 62:31-47. Medline

Kowler E, McKee SP (1987) Sensitivity of smooth eye movement to small differences in target velocity. Vision Res 27:993-1015. CrossRef Medline

Krauzlis RJ, Adler SA (2001) Effects of directional expectations on motion perception and pursuit eye movements. Vis Neurosci 18:365-376. CrossRef Medline

Krauzlis RJ, Lisberger SG (1994) A model of visually-guided smooth pursuit eye movements based on behavioral observations. J Comput Neurosci 1:265-283. CrossRef Medline

Linares D, Motoyoshi I, Nishida S (2012) Surround facilitation for rapid motion perception. J Vis 12: pii: 3. CrossRef Medline

Lisberger SG (2010) Visual guidance of smooth-pursuit eye movements: sensation, action, and what happens in between. Neuron 66:477-491. CrossRef Medline

Lisberger SG, Movshon JA (1999) Visual motion analysis for pursuit eye movements in area MT of macaque monkeys. J Neurosci 19:2224-2246. Medline

Lisberger SG, Westbrook LE (1985) Properties of visual inputs that initiate horizontal smooth pursuit eye movements in monkeys. J Neurosci 5:1662-1673. Medline
Lisberger SG, Evinger C, Johanson GW, Fuchs AF (1981) Relationship between eye acceleration and retinal image velocity during foveal smooth pursuit in man and monkey. J Neurophysiol 46:229-249. Medline

Lisberger SG, Morris EJ, Tychsen L (1987) Visual motion processing and sensory-motor integration for smooth pursuit eye movements. Annu Rev Neurosci 10:97-129. CrossRef Medline

Malone BJ, Ringach DL (2008) Dynamics of tuning in the Fourier domain. J Neurophysiol 100:239-248. CrossRef Medline

Maunsell JH, van Essen DC (1983) The connections of the middle temporal visual area (MT) and their relationship to a cortical hierarchy in the macaque monkey. J Neurosci 3:2563-2586. Medline

Morgan MJ (1992) Spatial filtering precedes motion detection. Nature 355: 344-346. CrossRef Medline

Morrone MC, Burr DC, Vaina LM (1995) Two stages of visual processing for radial and circular motion. Nature 376:507-509. CrossRef Medline

Movshon JA, Lisberger SG, Krauzlis RJ (1990) Visual cortical signals supporting smooth pursuit eye movements. Cold Spring Harb Symp Quant Biol 55:707-716. CrossRef Medline

Mukherjee T, Battifarano M, Simoncini C, Osborne LC (2015) Shared sensory estimates for human motion perception and pursuit eye movements. J Neurosci 35:8515-8530. CrossRef Medline

Mulligan JB (2002) Sensory processing delays measured with the eyemovement correlogram. Ann N Y Acad Sci 956:476-478. CrossRef Medline

Neri P, Levi D (2009) Surround motion silences signals from samedirection motion. J Neurophysiol 102:2594-2602. CrossRef Medline

Newsome WT, Paré EB (1988) A selective impairment of motion perception following lesions of the middle temporal visual area (MT). J Neurosci 8:2201-2211. Medline

Newsome WT, Wurtz RH, Dürsteler MR, Mikami A (1985) Deficits in visual motion processing following ibotenic acid lesions of the middle temporal visual area of the macaque monkey. J Neurosci 5:825-840. Medline

Orban GA, Van Essen D, Vanduffel W (2004) Comparative mapping of higher visual areas in monkeys and humans. Trends Cogn Sci 8:315-324. CrossRef Medline

Osborne LC, Lisberger SG (2009) Spatial and temporal integration of visual motion signals for smooth pursuit eye movements in monkeys. J Neurophysiol 102:2013-2025. CrossRef Medline

Osborne LC, Bialek W, Lisberger SG (2004) Time course of information about motion direction in visual area MT of macaque monkeys. J Neurosci 24:3210-3222. CrossRef Medline

Osborne LC, Lisberger SG, Bialek W (2005) A sensory source for motor variation. Nature 437:412-416. CrossRef Medline

Osborne LC, Hohl SS, Bialek W, Lisberger SG (2007) Time course of precision in smooth-pursuit eye movements of monkeys. J Neurosci 27:29872998. CrossRef Medline

Pack CC, Born RT (2001) Temporal dynamics of a neural solution to the aperture problem in visual area MT of macaque brain. Nature 409:1040 1042. CrossRef Medline

Papoulis A (1991) Probability, random variables and stochastic processes. New York: McGraw-Hill.

Peña JL, Konishi M (2001) Auditory spatial receptive fields created by multiplication. Science 292:249-252. CrossRef Medline

Rashbass C (1961) The relationship between saccadic and smooth tracking eye movements. J Physiol 159:326-338. CrossRef Medline

Raymond JE (1994) Directional anisotropy of motion sensitivity across the visual field. Vision Res 34:1029-1037. CrossRef Medline

Robinson DA (1963) A method of measuring eye movement using a scleral search coil in a magnetic field. IEEE Trans Biomed Eng 10:137-145. Medline

Rudolph K, Pasternak T (1999) Transient and permanent deficits in motion perception after lesions of cortical areas MT and MST in the macaque monkey. Cereb Cortex 9:90-100. CrossRef Medline

Sasaki R, Uka T (2011) Psychophysical evidence for contraction of the range of spatial integration as a mechanism for filtering out spatial noise in a random dot motion display. Vision Res 51:1979-1985. CrossRef Medline

Schütz AC, Braun DI, Movshon JA, Gegenfurtner KR (2010) Does the noise matter? Effects of different kinematogram types on smooth pursuit eye movements and perception. J Vis 10:26. Medline

Sheliga BM, Kodaka Y, FitzGibbon EJ, Miles FA (2006) Human ocular following initiated by competing image motions: evidence for a winnertake-all mechanism. Vision Res 46:2041-2060. CrossRef Medline

Sheliga BM, FitzGibbon EJ, Miles FA (2008) Human ocular following: evi- 
dence that responses to large-field stimuli are limited by local and global inhibitory influences. Prog Brain Res 171:237-243. CrossRef Medline

Sheliga BM, Fitzgibbon EJ, Miles FA (2008a) Spatial summation properties of the human ocular following response (OFR): evidence for nonlinearities due to local and global inhibitory interactions. Vision Res 48:17581776. CrossRef Medline

Sheliga BM, Fitzgibbon EJ, Miles FA (2009) The initial torsional Ocular Following Response (tOFR) in humans: a response to the total motion energy in the stimulus? J Vis. 9:2.1-38. Medline

Sincich LC, Horton JC, Sharpee TO (2009) Preserving information in neural transmission. J Neurosci 29:6207-6216. CrossRef Medline

Smith AT, Snowden RJ, Milne AB (1994) Is global motion really based on spatial integration of local motion signals? Vision Res 34:2425-2430. CrossRef Medline

Stocker AA, Simoncelli EP (2006) Noise characteristics and prior expectations in human visual speed perception. Nat Neurosci 9:578-585. CrossRef Medline

Stone LS, Krauzlis RJ (2003) Shared motion signals for human perceptual decisions and oculomotor actions. J Vis 3:725-736. Medline

Sun D, Roth S, Black MJ (2010) Secrets of optical flow estimation and their principles. Proc IEEE Conf Computer Vision and Pattern Recognition 2432-2439. CrossRef

Tadin D, Lappin JS (2005) Optimal size for perceiving motion decreases with contrast. Vision Res 45:2059-2064. CrossRef Medline

Tadin D, Lappin JS, Gilroy LA, Blake R (2003) Perceptual consequences of centre-surround antagonism in visual motion processing. Nature 424: 312-315. CrossRef Medline

Tadin D, Lappin JS, Blake R (2006) Fine temporal properties of centersurround interactions in motion revealed by reverse correlation. J Neurosci 26:2614-2622. CrossRef Medline

Tadin D, Paffen CL, Blake R, Lappin JS (2008) Contextual modulations of center-surround interactions in motion revealed with the motion aftereffect. J Vis. 8:9.1-11. Medline
Tavassoli A, Ringach DL (2009) Dynamics of smooth pursuit maintenance. J Neurophysiol 102:110-118. CrossRef Medline

van Doorn AJ, Koenderink JJ (1984) Spatiotemporal integration in the detection of coherent motion. Vision Res 24:47-53. CrossRef Medline

Verghese P, Watamaniuk SN, McKee SP, Grzywacz NM (1999) Local motion detectors cannot account for the detectability of an extended trajectory in noise. Vision Res 39:19-30. CrossRef Medline

Watamaniuk SN, Heinen SJ (1999) Human smooth pursuit direction discrimination. Vision Res 39:59-70. CrossRef Medline

Watamaniuk SN, Sekuler R (1992) Temporal and spatial integration in dynamic random-dot stimuli. Vision Res 32:2341-2347. CrossRef Medline

Watamaniuk SN, Sekuler R, McKee SP (2011) Perceived global flow direction reveals local vector weighting by luminance. Vision Res 51:11291136. CrossRef Medline

Watamaniuk SN, Sekuler R, Williams DW (1989) Direction perception in complex dynamic displays: the integration of direction information. Vision Res 29:47-59. CrossRef Medline

Watson AB, Turano K (1995) The optimal motion stimulus. Vision Res 35:325-336. CrossRef Medline

Weiner N (1949) Extrapolation, interpolation, and smoothing of time series. New York: Wiley.

Williams DW, Sekuler R (1984) Coherent global motion percepts from stochastic local motions. Vision Res 24:55-62. CrossRef Medline

Wilmer JB, Nakayama K (2007) Two distinct visual motion mechanisms for smooth pursuit: evidence from individual differences. Neuron 54:9871000. CrossRef Medline

Womelsdorf T, Anton-Erxleben K, Treue S (2008) Receptive field shift and shrinkage in macaque middle temporal area through attentional gain modulation. J Neurosci 28:8934-8944. CrossRef Medline

Yang J, Lee J, Lisberger SG (2012) The interaction of bayesian priors and sensory data and its neural circuit implementation in visually guided movement. J Neurosci 32:17632-17645. CrossRef Medline 